\title{
Characterization of the Contractive and Pore Pressure Behavior of Saturated Sand Deposits under Seismic Loading
}

\author{
Vicente Mercado (Corresponding Author) \\ Assistant Professor, Universidad del Norte, Km. 5 Vía a Puerto Colombia, Of. 8-21K, Barranquilla, Colombia. Email: \\ vmercado@uninorte.edu.co. \\ Waleed El-Sekelly \\ Assistant Professor, Mansoura University, Mansoura, Egypt. Email: waleed.elsekelly@ gmail.com \\ Mourad Zeghal \\ Professor, Rensselaer Polytechnic Institute, 110 8th Street, Troy, NY 12180. Email: zeghal@ rpi.edu \\ Tarek Abdoun \\ Iovino Chair Professor, Rensselaer Polytechnic Institute, 110 8th Street, Troy, NY 12180. Email: abdout@ rpi.edu \\ Ricardo Dobry \\ Institute Professor, Rensselaer Polytechnic Institute, 110 8th Street, Troy, NY 12180. Email: dobryr@ rpi.edu
}

Sabanayagam Thevanayagam

Professor, University of Buffalo, 212 Ketter Hall, Buffalo, NY 14260. Email: theva @ buffalo.edu

\begin{abstract}
This paper presents an identification technique to characterize the contractive and pore pressure behavior of loose sandy soils under seismic excitation. The technique relies on acceleration and pore pressure records provided during excitation by vertical arrays of accelerometers and pore pressure sensors. The technique employs non-parametric estimates of shear stresses and strains. A multi-surface plasticity approach is used to model the soil response. A reduced-scale centrifuge model and a large scale experiment are used to demonstrate the capabilities of the developed technique. The technique allows for a more complete interpretation of the coupled shear-volume behavior of a soil deposit.
\end{abstract}

\section{Keywords}

System identification; Downhole array; Soil dynamics; Pore pressure response 


\section{Introduction}

Earthquake induced liquefaction of sandy soils has been the cause of severe damage to buildings and other structures all over the world, as shown again by recent earthquakes in Haiti (2010), Chile (2015), Japan (2011) and New Zealand (2010). Earthquake shaking generates cyclic shear loading which leads loose sand to contract, resulting in a transfer of normal stresses from sand grains to pore water. This is particularly true if the sand is fully saturated and unable to drain rapidly during shaking. This results in an overall reduction in the effective stress and hence, reduction in the shear stiffness and shear strength of the sand resulting in large deformation of the soil deposit and the damage or even destruction of supported structures [1]. Extensive efforts have been exerted by researchers and practitioners trying to predict the liquefaction behavior of sandy deposits for the purpose of assessment of safety and integrity of new and existing structures.

Along these lines, several researchers have developed a variety of system identification techniques in order to characterize the dynamic soil behavior and the associated pore pressure response. These developments have been possible due to the recent availability of high quality seismic records of sites equipped with vertical (downhole) arrays of accelerometers and pore-pressure sensors (e.g., [2], [3], [4], [5], [6]). The current state-of-the-art in centrifuge model testing and large scale testing of soil deposits also relies on vertical arrays of acceleration and pore-pressure sensors (e.g., [7], [8], [9], [10], [11]).

Developed inverse analysis techniques for identification of dynamic soil behavior include efforts by Zeghal et al. [2], [12] for the direct evaluation of non-parametric 
estimates of shear stresses and strains using the accelerations provided by vertical (downhole) arrays. Additionally, Assimaki et al. [5], presented a full waveform inversion algorithm of downhole array seismogram recordings to estimate the inelastic behavior of soil deposits during earthquake ground motion. This work used a global optimization scheme to estimate low-strain soil properties of instrumented sites [13], [14], [15]. Tsai and Hashash [16], [17] introduced a self-learning inverse analysis algorithm (SelfSim), that can learn and extract soil behavior from recorded events using neural networks.

Downhole array data has also been used to explore the pore pressures generation during earthquake events, and to evaluate the validity of site response analysis models. Matasovic [18], for instance, evaluated the performance of the computer program DMOD in predicting site response and pore pressure generation using data from the Imperial Valley Wildlife Liquefaction Array. Yang and Elgamal [19] applied an optimization analysis for the calibration of a multi-surface plasticity soil model that handles the coupling effects of soil behavior and pore pressure buildup. More recently, Groholski and Hashash [20], and Groholski et al. [6] extended the SelfSim framework to effective-stress considerations in order to extract both soil behavior and pore pressure response from recorded motions and pore pressures during seismic events.

This paper presents a technique to identify the coupled shear-volume response of sand deposits that can lead to significant generation of pore water pressures. The technique incorporates pore pressure records, as well as shear stress and strain estimates of a soil deposit subjected to dynamic excitation, along with a multi-surface constitutive model. The selected material model, based on previous models presented by Prevost [21], and Yang and Elgamal [19], is capable of handling the cyclic mobility response mechanism and associated pore pressure buildup of soils. This model controls the contractive response of 
the soil through a single calibration constant, which can be adjusted in order to capture the coupled volumetric response induced by shear loading. The proposed identification technique is first tested for convergence and accuracy using numerical simulations. The technique is then applied to experimental data from a centrifuge test and a large scale experiment.

\section{System identification approach}

The employed identification technique uses non-parametric estimates of shear stress and strain histories obtained directly from vertical arrays of acceleration records, and recorded excess pore pressure histories, to estimate a constitutive parameter defining the contractive behavior of the associated soil. The developed local identification technique is briefly described below:

(1) Shear stress and strain histories estimates are obtained using a methodology proposed by Zeghal, Elgamal and co-workers [2], [12]. This methodology allows for the direct evaluation of non-parametric estimates of the associated shear stresses and strains at several depth locations using the accelerations provided by vertical (downhole) arrays under conditions of vertical shear wave propagation.

(2) Estimated strain time histories are used along with a constitutive (stress-strain) model to evaluate the corresponding (parametric) stress response.

(3) An objective function is evaluated based on discrepancies between the computed stress response and the recorded behavior of the soil as follows: shear stresses predicted by the employed soil model are compared to shear stresses estimated from acceleration records, and the reduction of the modeled effective vertical stress is compared to the recorded increase in excess pore water pressure. 
(4) An optimization algorithm is implemented to determine optimal values of a constitutive parameter (defining the contractive behavior of the soil) that minimizes the objective function.

The implemented stress-strain constitutive model calculates the stress response using the prescribed (non-parametric) strain time history. This is advantageous as it does not require the forward modeling of the whole soil deposit. Even though the model does not deal with the pore pressure response directly, the reduction in the modeled effective vertical stress obtained for a given shear strain time history should coincide with the increase in pore water pressure that the corresponding actual soil deposit would experience for the same strains (this is further discussed within the context of the numerical simulations in Section 3). In practice, however, vertical strains at different depths of the soil deposit are difficult to measure and this information is often not available. Because of this, vertical strains are assumed to be zero in the presented identification analysis, thus ignoring the volumetric changes that take place due to water flow and consolidation during the shaking that typically occurs during seismic excitation. This may result in some bias in the identified parameters, especially for very high permeability soils. Nevertheless, the conducted identification analyses for soils with a permeability comparable to that of sandy soil showed that the employed technique leads to a reasonably good agreement with the soil actual contractive parameters.

In this paper, the shear stress-strain relation of the soil profile in the absence of pore pressure buildup is assumed to be a priori known. This relation can be expressed in terms of a small-strain shear modulus, $G_{\max }$, and reference shear deformation, $\gamma_{\text {ref }}$. A methodology for the estimation of these parameters was previously proposed by the authors [22]. 
Additionally, values of $G_{\max }$ can be inferred from direct shear wave velocity measurements, if these are available.

It should be also mentioned that the technique does not require the forward modeling of the whole soil deposit, since the analysis can be performed for a particular depth of the deposit using estimations of shear stresses, shear strains, and excess pore pressure at that particular depth. The identification process is shown schematically in Fig. 1. The different components of the algorithm are explained in the following sections.

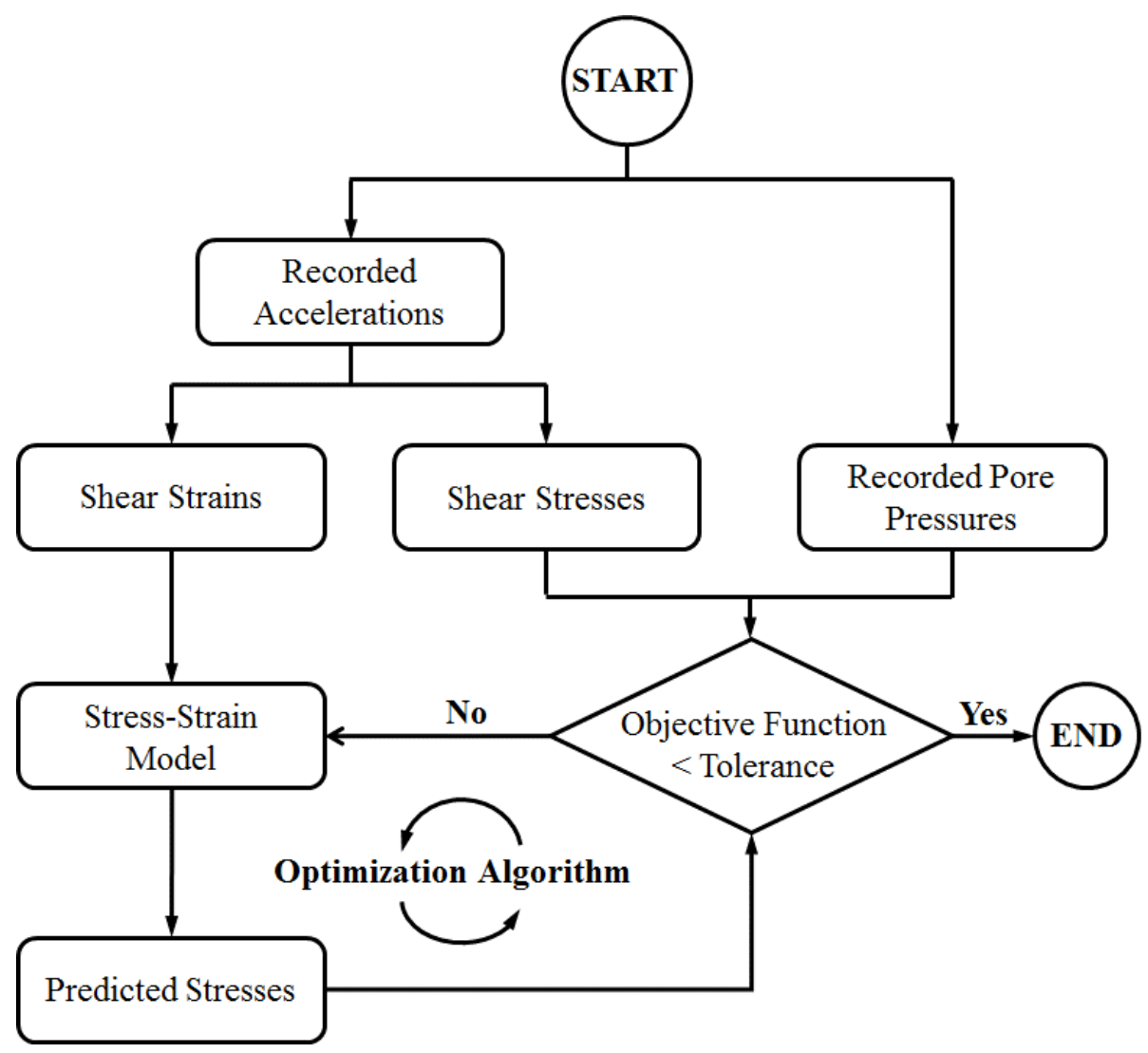

Fig. 1. Schematic of the employed identification algorithm. 


\subsection{Stress and strain estimates}

Zeghal, Elgamal and co-workers [2], [12] proposed a methodology for the estimation of shear stress and strain time histories based on acceleration records provided by a downhole array (Fig. 2). A one-dimensional shear beam idealization is used to describe the lateral response of the deposit along a vertical array: $\partial \tau / \partial z=\rho \ddot{\boldsymbol{u}}$, with boundary condition $\tau(0, t)$ $=0$, where $t$ is time, $z$ is depth, $\boldsymbol{\tau}=\boldsymbol{\tau}(z, t)=\left\{\tau_{z x}(z, t), \tau_{z y}(z, t)\right\}$ is the horizontal shear stress vector, and $\ddot{\boldsymbol{u}}=\ddot{\boldsymbol{u}}(z, t)=\left\{\ddot{u}_{x}(z, t), \ddot{u}_{y}(z, t)\right\}$ is the horizontal acceleration vector.

Integrating the equation of motion and using a stress free surface boundary the shear stress at any level $z$ may be evaluated using. :

$$
\boldsymbol{\tau}(z, t)=\int_{0}^{z} \rho \ddot{\boldsymbol{u}} d z
$$

Discrete expressions for shear stresses at certain depths may be derived employing linear interpolation between downhole accelerations. These stress estimates are second-order accurate [3].

The corresponding shear strain is given by:

$$
\boldsymbol{\gamma}=\partial \boldsymbol{u} / \partial z
$$

, where $\boldsymbol{\gamma}=\left\{\gamma_{z x}(z, t), \gamma_{z y}(z, t)\right\}$, and $\boldsymbol{u}=\left\{u_{x}(z, t), u_{y}(z, t)\right\}$ is displacement vector. Second-order accurate shear-strain estimates at certain depth locations may be derived from linear interpolations [3]. 


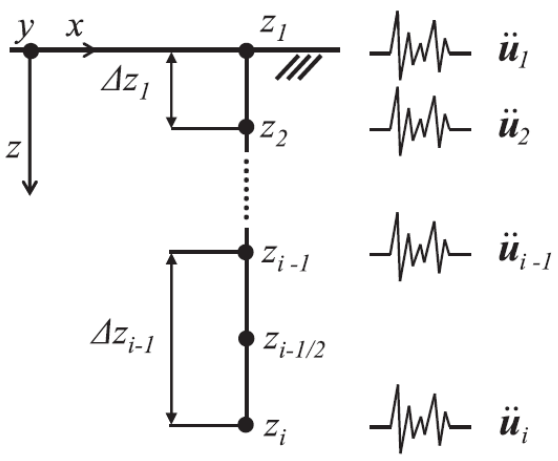

Fig. 2. Schematic array of accelerometers locations (after [22]).

\subsection{Soil stress-strain model}

A multi-surface plasticity technique is used to idealize the nonlinear and path dependent stress-strain soil response [23], [24]. Relevant aspects of the model are outlined below. More details of the multi-surface plasticity models may be found elsewhere (e.g., [21], [23], $[25])$.

The model assumes that the total strain rate $\dot{\boldsymbol{\varepsilon}}$ can be expressed as the sum of elastic $\dot{\boldsymbol{\varepsilon}}^{e}$ and plastic $\dot{\boldsymbol{\varepsilon}}^{p}$ strain rates; therefore the constitutive equation is written as:

$$
\dot{\boldsymbol{\sigma}}^{\prime}=\boldsymbol{E}:\left(\dot{\boldsymbol{\varepsilon}}-\dot{\boldsymbol{\varepsilon}}^{p}\right)
$$

where $\boldsymbol{\sigma}^{\prime}$ is the effective (Cauchy) stress tensor, $\boldsymbol{E}$ is the isotropic fourth-order tensor of elastic coefficients, and ":" denotes a second order dyadic product of two tensors.

The plastic rate of deformation, which gives the direction of the plastic component of the strain rate, is defined as:

$$
\dot{\boldsymbol{\varepsilon}}^{p}=\langle\lambda\rangle \boldsymbol{P}
$$


where $\boldsymbol{P}$ is a symmetric second-order tensor which defines the direction in stress space of plastic deformations, $\lambda$ is the plastic loading function, and the symbol \langle\rangle denotes the MacCauley's bracket: $\langle\lambda\rangle=\lambda$ if $\lambda \geq 0$; otherwise $\langle\lambda\rangle=0$.

The plastic loading function is defined as:

$$
\lambda=\frac{1}{H^{p}} \boldsymbol{Q}: \dot{\boldsymbol{\sigma}}^{\prime}
$$

where $Q$ is a second order symmetric tensor denoting the outer normal to the yield surface, and $H^{p}$ is the plastic modulus.

\subsubsection{Yield function}

The strain hardening of the shear stress-strain relationship is handled using a yield function which forms a conical surface in the principal stress space, with its apex along the hydrostatic axis. The function is given by Prevost [21]:

$$
f=\frac{3}{2}\left(s-p^{\prime} \boldsymbol{\alpha}\right):\left(s-p^{\prime} \boldsymbol{\alpha}\right)-k^{2} p^{\prime 2}=0
$$

where $p^{\prime}$ is the mean effective stress, $\boldsymbol{s}=\boldsymbol{\sigma}^{\prime}-p^{\prime} \boldsymbol{\delta}$ is the deviatoric stress tensor (with $\boldsymbol{\delta}=$ second-order identity tensor), $\boldsymbol{\alpha}$ is a tensor indicating the coordinates of the center of the

yield surface in the deviatoric stress subspace, and $k$ is a parameter that defines the size of the yield surface. 


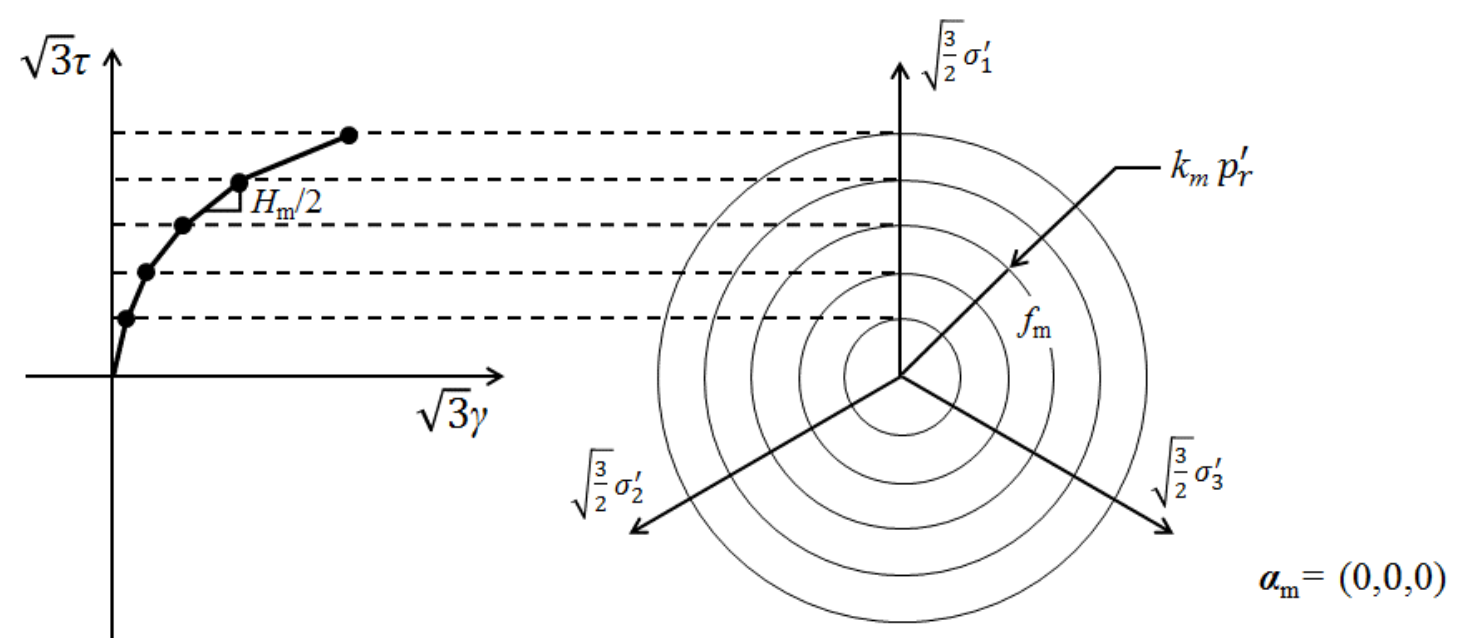

Fig. 3. Relationship between the backbone curve and initial parameters of the nested yield surfaces on a deviatoric stress plane (after [22], [24], [25]).

A hyperbolic function [26] was used to define the soil nonlinear stress-strain backbone curve at a given reference confinement $p_{r}^{\prime}$. This function is simpler and more widely used than other stress-strain relations (e.g., [27], [28]), and is given by:

$$
\tau(\gamma)=\frac{G_{\max } \gamma}{1+\frac{\gamma}{\gamma_{\text {ref }}}}
$$

where $\tau$ and $\gamma$ are shear stress and strain amplitudes respectively, $G_{\text {max }}$ is the small-strain shear modulus at $p_{r}^{\prime}, \gamma_{\text {ref }}=\tau_{\max } / G_{\max }$ is a reference deformation [29], and $\tau_{\max }$ is the shear strength of the soil at $p_{r}^{\prime}$. The stiffness of the material is assumed to vary with the effective mean confining stress as follows:

$$
G=G_{\max }\left(\frac{p^{\prime}}{p_{r}^{\prime}}\right)^{0.5}
$$


where $G$ is the small-strain shear modulus at $p^{\prime}[21]$.

Within the context of multi-surface plasticity, a collection of nested yield surfaces is introduced in order to allow for the adjustment of the plastic hardening rule to a variety of experimental or theoretical data. The definition of each of these yield surfaces is given by $f_{m}=\frac{3}{2}\left(\boldsymbol{s}-p^{\prime} \boldsymbol{\alpha}_{m}\right):\left(\boldsymbol{s}-p^{\prime} \boldsymbol{\alpha}_{m}\right)-k_{m}{ }^{2} p^{2}=0$, where the subscript $m$ refers to the $m^{\text {th }}$ yield surface. This collection of yield surfaces allows for a piecewise linear approximation of the hyperbolic backbone curve of the material. Each surface $f_{m}$ is associated with a (tangent) shear modulus $H_{m}$, and the size of the surface $k_{m}$. The parameters $k_{m}$ and $H_{m}$, and the initial values of $\boldsymbol{\alpha}_{m}$ are dictated by the shape of the shear stress-strain "backbone" curve, as shown in Fig. 3 [24], [25]. The tangent shear modulus is assumed to follow the same confinement dependence as the shear modulus (Eq. 8) [21].

\subsubsection{Flow rule}

An associated flow rule was used to model the plastic behavior of the deviatoric components of the stress-strain behavior. That is, the deviatoric component of the direction tensor $\boldsymbol{P}$ is defined as equal to the deviatoric component of tensor $\boldsymbol{Q}$. The volumetric plastic flow, on the other hand is selected as non-associative, in order to correctly model the volume change [21], [30]. More specifically, the volumetric component $P^{\prime \prime}$ of the plastic flow direction tensor is given as:

$$
3 P^{\prime \prime}=c_{1} \frac{\left(\eta / \eta_{P T}\right)^{2}-1}{\left(\eta / \eta_{P T}\right)^{2}+1}
$$

where $\eta=\sqrt{3 / 2(s: s)} / p^{\prime}$ is stress ratio, $\eta_{P T}$ is the stress ratio along the phase transformation surface, and $c_{1}$ is a contration constant which adds flexibility in adjusting 
the volumetric response. Under undrained conditions, a larger $c_{1}$ represents a larger contractive tendency in the material, resulting in a higher pore water pressure buildup rate, and a consequent reduction in stiffness and strength. Figure 4, for instance, illustrates the modeled shear stress-strain behavior, and shear stress-effective confinement relationship for strain-controlled undrained loading for two values of $c_{1}$. It is observed that a larger $c_{1}$ results in greater contractive tendency in the material for the same number of loading cycles, accompanied by a larger reduction of the effective mean confining stress.

It has been established that the phase transformation surface indicates the stress ratio at which the behavior of the soil transitions from contractive to dilative [31]. This paper considers only the contractive phase of the behavior of soils and analyses are performed only for soils experiencing stress states that do not surpass $\eta_{P T}$. Consequently, these soils do not experience a dilative tendency. Since the analyzed values of $\eta$ never reach the value of $\eta_{P T}$, it is assumed, for simplicity, that the value of $\eta_{P T}$ coincides with the stress ratio corresponding to the shear strength of the soil. 

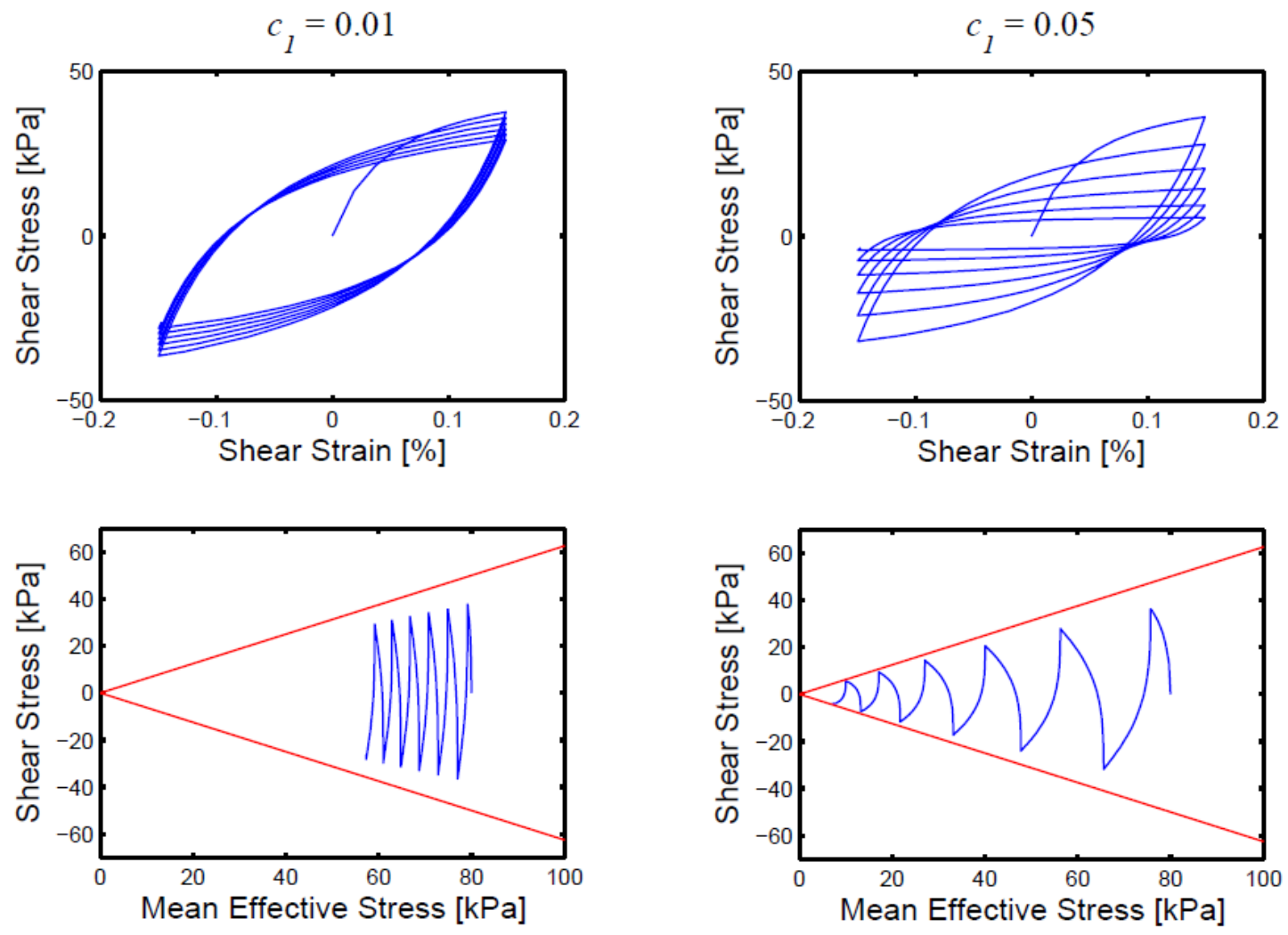

Fig. 4. Shear stress-strain response, and shear stress-effective confinement relationship for strain-controlled undrained loading for two values of $c_{1}(0.01$ and 0.05$)$.

\subsubsection{Hardening rule}

A purely deviatoric kinematic hardening rule is adopted (yield surfaces are assumed to translate, but not to change in size or orientation). When the stress point reaches a yield surface, as it moves towards the next yield surface, all previous yield surfaces are translated together by the stress point until the new stress point is reached. This translation enables a hysteretic and path dependent stress-strain response. The translation of the yield surfaces is defined by the following relation $\dot{\boldsymbol{\alpha}}=\langle\lambda\rangle A \boldsymbol{\mu}$, where $\boldsymbol{\mu}$ is the deviatoric tensor defining the direction of translation of the yield surface, and $A$ is the amount of translation determined through the consistency condition and may be expressed as: 


$$
A=\frac{H^{p}}{Q: \mu}
$$

In order to avoid overlapping of the surfaces (so that the plastic loading function be uniquely defined), the surfaces must remain tangent to each other at the stress point. Further, contact between $f_{m}$ and $f_{m+1}$ must occur at points with the same direction of outwards normal, as illustrated in Fig. 5 [21].

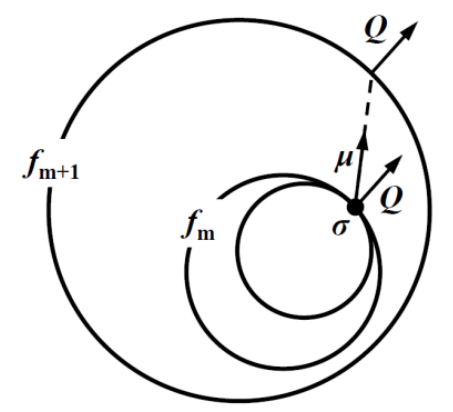

Fig. 5. Definition of translation direction (after [21]).

\subsection{Parameter Identification}

A least squares algorithm was employed to identify the contractive behavior of the soil expressed in terms of the contraction parameter $c_{1}$. The associated objective function, $O\left(c_{1}\right)$, is expressed as a measure of the error between the recorded behavior and the modeled response. More specifically shear stresses predicted by the employed soil model are compared to shear stresses estimated from acceleration records, and the reduction of the modeled effective vertical stress is compared to the recorded increase in excess pore water pressure, as follows: 


$$
O\left(c_{1}\right)=\sum_{t=t_{i}}^{t_{f}}\left[\left(\boldsymbol{\tau}^{(r)}(t)-\boldsymbol{\tau}^{(m)}(t)\right)^{2}+W\left(\Delta u_{w}(t)-\left|\Delta \sigma_{v}^{\prime}(t)\right|\right)^{2}\right]
$$

in which $\boldsymbol{\tau}^{(r)}(t)$ is the vector of shear stress components estimated from acceleration records at time $t$ (Eq. 1), $\boldsymbol{\tau}^{(m)}(t)$ is the shear stress vector at time $t$ predicted by the implemented material model along with estimated cyclic shear strains, $\Delta u_{w}(t)$ is the recorded excess pore pressure, $\left|\Delta \sigma_{v}^{\prime}(t)\right|=\left|\sigma_{v}^{\prime}(t)-\sigma_{v o}^{\prime}\right|$ is the absolute value of the change in the vertical effective stress at time $t$ predicted by the material model (with $\sigma_{v o}^{\prime}$ as the initial effective vertical stress, and $\sigma_{v}^{\prime}(t)$ as effective vertical stress at time $\left.t\right),\left[t_{i}, t_{f}\right]$ is the time window selected for the identification analysis, and $W$ is an adjustable weighing factor. The optimal model parameter $c_{1}$ is obtained by minimization of the objective function.

In principle, the identification problem could be resolved using either of the two terms in the objective function, and for the subsequent analyses the value of $W$ did not have great incidence over the identification results. However values of $W$ were selected so that the two terms in the objective function contribute similar magnitudes [19].

In this study, the optimization analysis was performed using the nonlinear least squares code lsqnonlin, of the Optimization Toolbox by MathWorks [32]. The associated algorithm employs a steepest descent approach to minimize the objective function.

\section{Computer simulations and verification}

Computer simulations were used to assess the capabilities of the developed identification technique. A finite element computational model was used to generate synthetic seismic records of the $7 \mathrm{~m}$ saturated soil (level) deposit depicted in Fig. 6. Following modeling 
practices suggested by Elgamal et al. [33], the surface was traction free, with zero prescribed pore pressure, and the base and lateral boundaries were impervious. At any given depth, displacement degrees of freedom were tied together (both horizontally and vertically). Gravity was applied statically leading to static hydrostatic pressures and soil stress-states along the soil column. These served as initial conditions for the subsequent application of a dynamic excitation.

The soil in the deposit was characterized by the constitutive model presented in the previous section, using a distribution of material parameters typical of a uniform deposit of a cohesionless soil. Modeling parameters included: a small-strain shear modulus $G_{\max }$ of $150 \mathrm{MPa}$ (at $80 \mathrm{kPa}$ reference mean effective confinement $p_{r}^{\prime}$ ), maximum shear stress corresponding to a friction angle of $30^{\circ}$, and a permeability of $1 \times 10^{-5} \mathrm{~m} / \mathrm{s}$. In order to avoid dilation, the phase transformation angle is assumed to coincide with the angle of friction. The deposit was subjected to 5 seconds of a uniform sinusoidal acceleration with a $2 \mathrm{~Hz}$ frequency and a peak amplitude of $0.08 \mathrm{~g}$. Two cases were simulated for the subsequent analyses: (a) Case 1, which uses contraction parameter $c_{1}=0.02$ for the whole deposit, and (b) Case 2 which uses $c_{1}=0.1$. The accelerations at the base and at selected locations within the deposits are displayed in Fig. 7. 


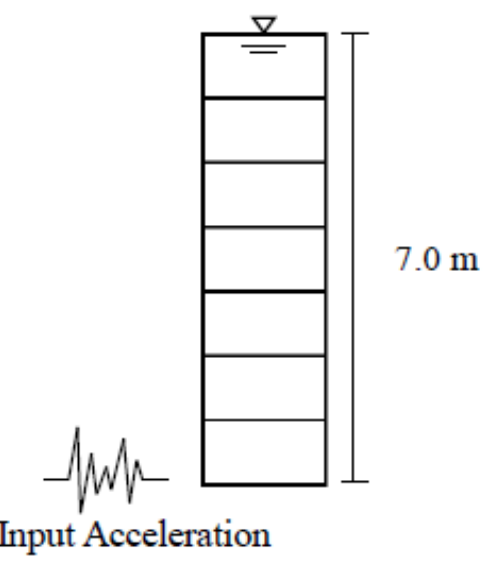

Fig. 6. Configuration of analyzed finite element computational model of a soil deposit.
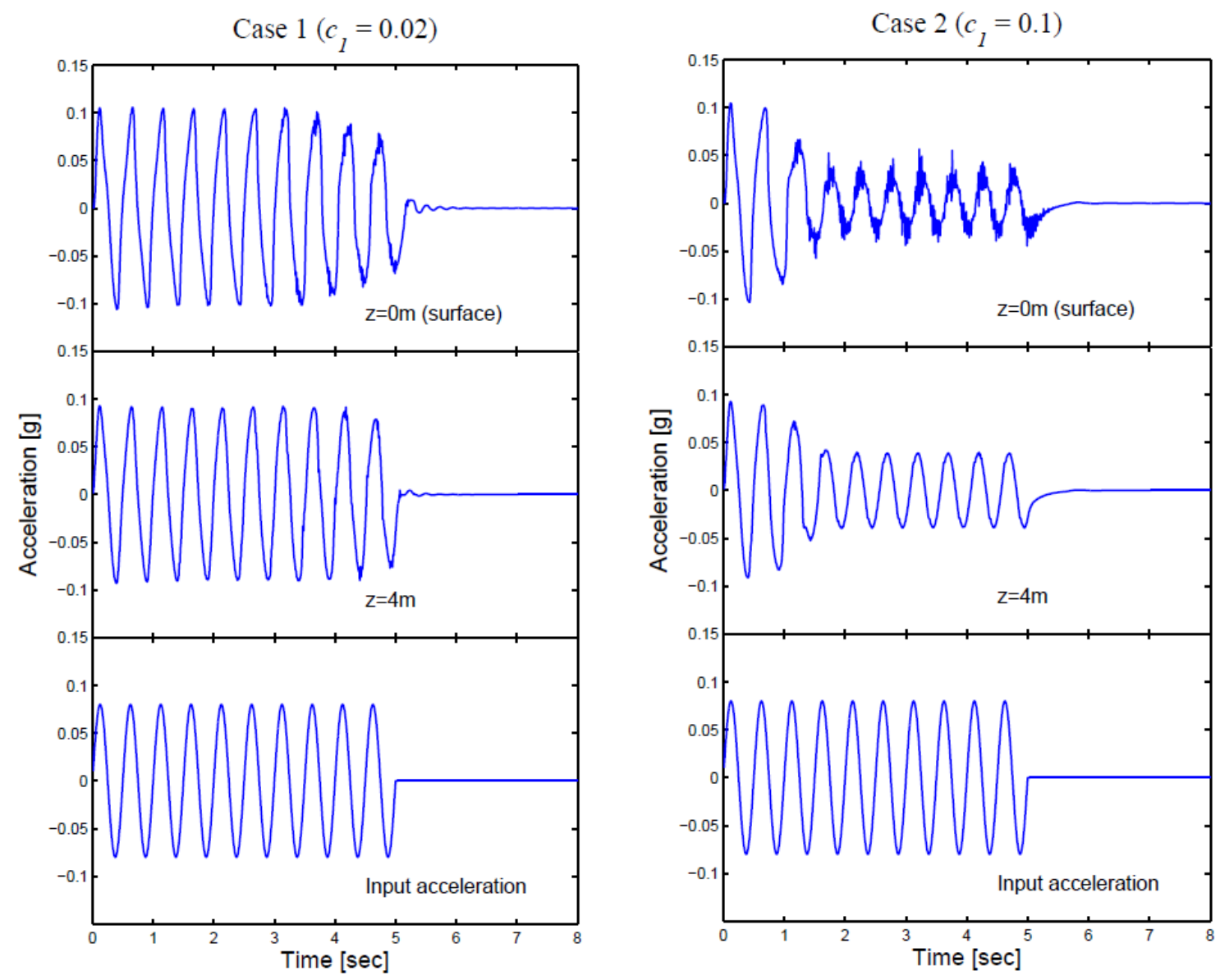

Fig. 7. Input and recorded soil accelerations at selected depth locations for the computational simulations using $c_{1}=0.02$ and $c_{1}=0.1$. 
The simulated tests exhibited very different behaviors, illustrating the importance of the value of $c_{1}$. Case 1 showed a relatively slow increase in excess pore water pressure, accompanied by a slow and relatively small reduction in the amplitude of the shear stress peaks (Figs. 8 and 9). Case 2, on the other hand, showed a rapid increase in excess pore water pressure, accompanied by an abrupt reduction of the shear stress peaks (Figs. 8 and 9). This is consistent with the values of $c_{1}$ that were used for the simulations $\left(c_{1}=0.02\right.$ for Case 1 , and $c_{1}=0.1$ for Case 2). Identification analyses were conducted for the two cases at two different depths $(z=1.5 \mathrm{~m}$ and $z=5.5 \mathrm{~m})$ using the synthetic strain response of the finite element model. These synthetic strain time histories were used along the material model and a trial value of the contraction parameter $c_{1}$ to generate a prediction of the stresses. As mentioned earlier, vertical strains introduced to the constitutive model used in the identification are defined as zero in order to replicate limitations of the analyses when dealing with actual field data in which vertical strain information is not available. The stresses predicted by the material model $\left(\boldsymbol{\tau}^{(m)}(t)\right.$ and $\left.\left|\Delta \sigma_{v}^{\prime}(t)\right|\right)$ are compared to the finite element model's synthetic shear stress and excess pore pressure response $\left(\boldsymbol{\tau}^{(r)}(t)\right.$ and $\left.\Delta u_{w}(t)\right)$ using the formulation of the objective function of Eq. 11. Minimization of the objective function $O\left(c_{1}\right)$ leads to the identified contraction parameters $c_{1}$ summarized in Table 1 and depicted in Fig. 10. An investigation of the variation of the objective function $O\left(c_{1}\right)$ as a function of $c_{1}$ for the performed analyses confirms that there is a unique solution to the identification problem, as shown in Fig. 11. The functions exhibit a smooth curve with a well-defined minimum, and a reasonable sensitivity to the contraction parameter, which allows for an adequate optimization using gradient-based methods. 

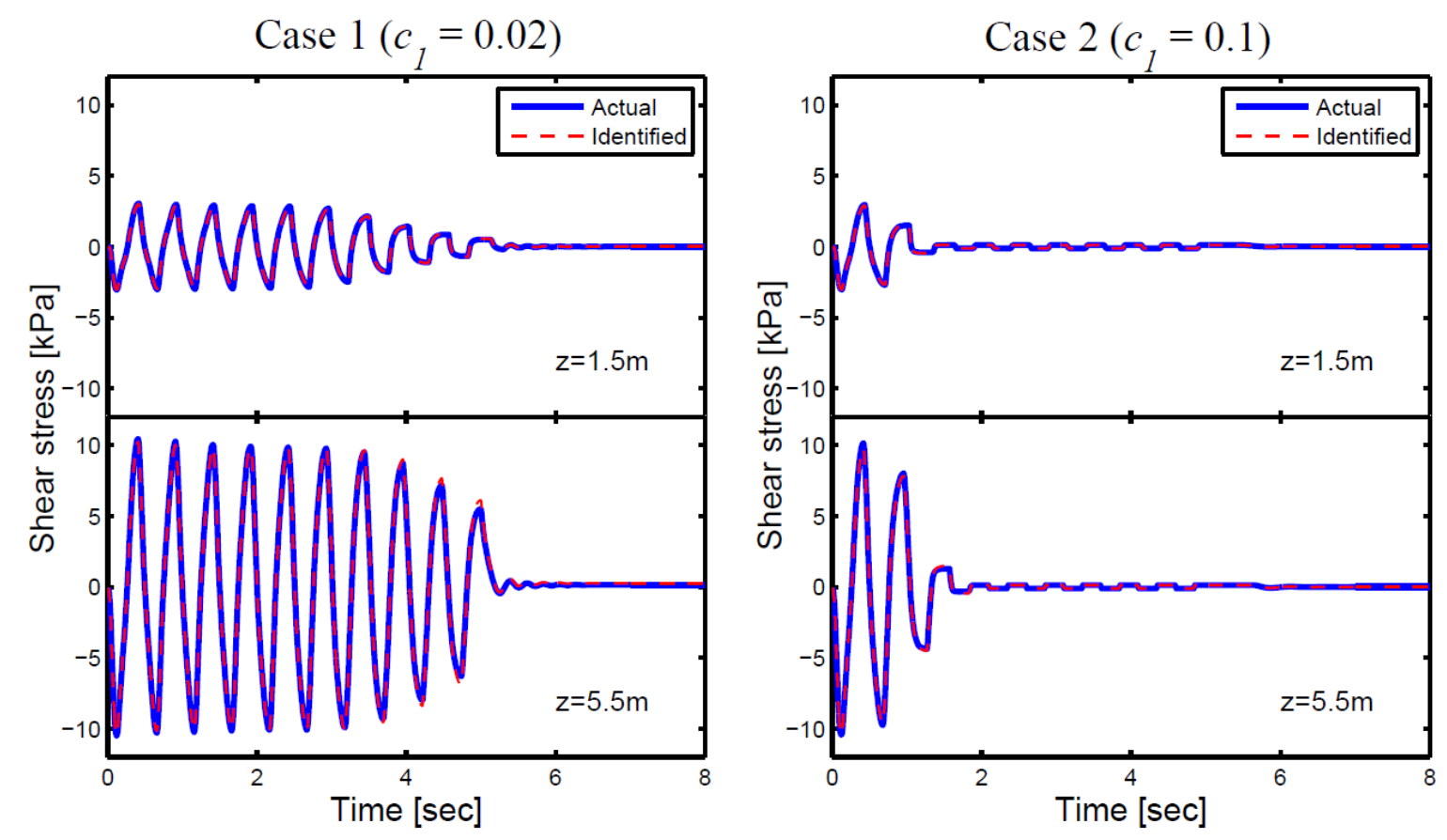

Fig. 8. Shear stress time histories obtained from the computer simulation along with those obtained using the identified contraction parameter $c_{1}$ for Case 1 and Case 2. 

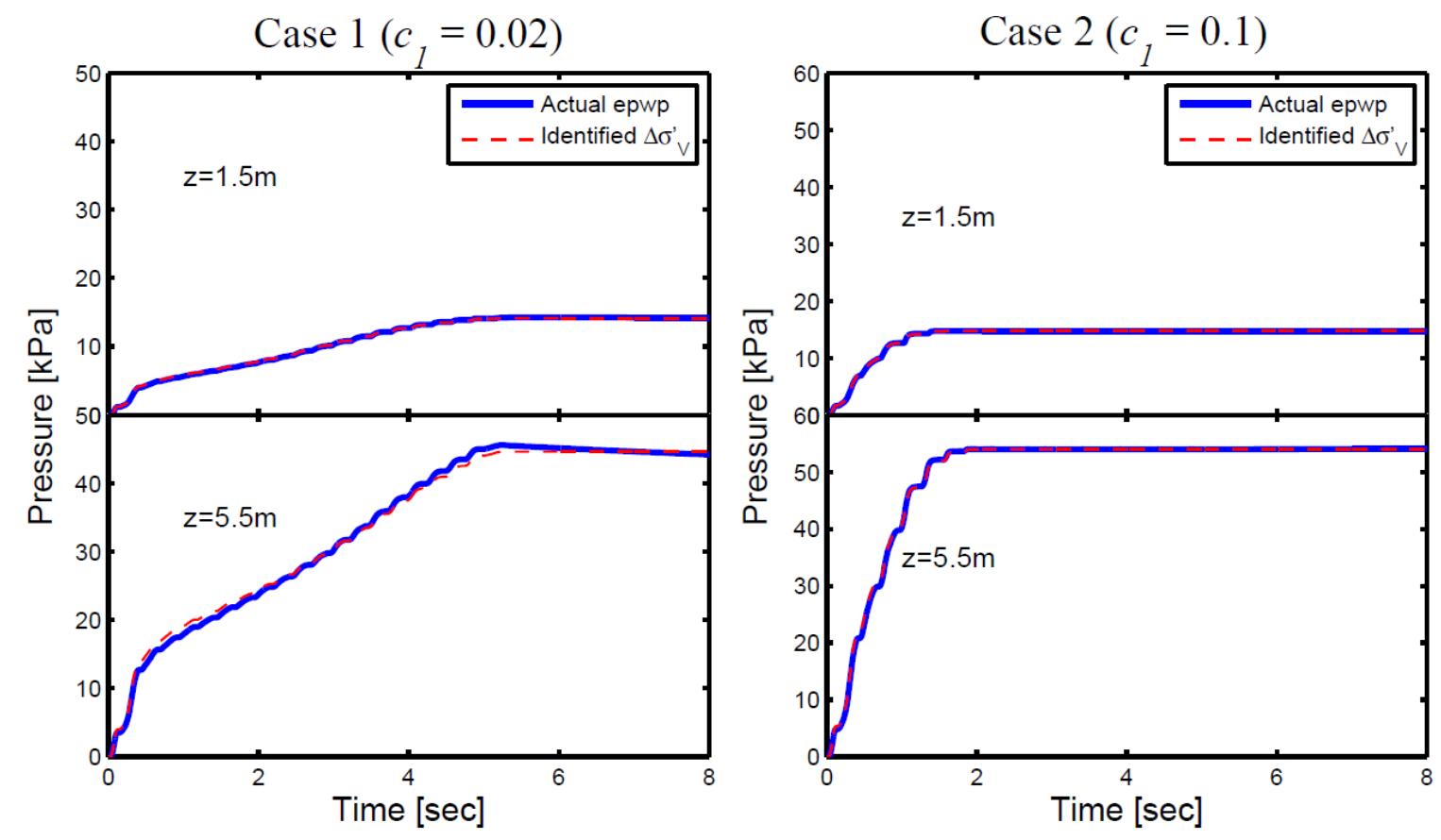

Fig. 9. Excess pore water pressure (epwp) obtained from the computer simulation along with absolute value of the change in effective vertical stress $\left(\Delta \sigma^{\prime}{ }_{v}\right)$ obtained using the identified contraction parameter $c_{1}$ for Case 1 and Case 2 .

Figure 8 shows very close agreement between the actual (synthetic) shear stress time histories of the computational simulation along with those predicted by the material model using the identified material parameters. The relative error between synthetic and predicted shear stresses is given by:

$$
E_{\tau}=\left[\sum_{t=t_{i}}^{t_{f}}\left(\boldsymbol{\tau}^{(r)}(t)-\boldsymbol{\tau}^{(m)}(t)\right)^{2} / \sum_{t=t_{i}}^{t_{f}}\left(\boldsymbol{\tau}^{(r)}(t)\right)^{2}\right]^{1 / 2}
$$

For Case 1 the relative error $E_{\tau}$ was $1.9 \%$ at $1.5 \mathrm{~m}$ and $3.7 \%$ at $5.5 \mathrm{~m}$, and for Case 2 it was $2.2 \%$ at $1.5 \mathrm{~m}$ and $2.9 \%$ at $5.5 \mathrm{~m}$. Actual (synthetic) excess pore pressures of the computational simulation are also in very close agreement with the magnitudes of the 
change in vertical effective stress, $\left|\Delta \sigma_{v}^{\prime}\right|$, predicted by the material model using the identified parameters, as shown in Fig. 9. The relative error between synthetic excess pore pressure, and the predicted change in vertical effective stress is given by:

$$
E_{\sigma}=\left[\sum_{t=t_{i}}^{t_{f}}\left(\Delta u_{w}(t)-\left|\Delta \sigma_{v}^{\prime}(t)\right|\right)^{2} / \sum_{t=t_{i}}^{t_{f}}\left(\Delta u_{w}(t)\right)^{2}\right]^{1 / 2}
$$

For Case 1 the relative error $E_{\sigma}$ was $0.8 \%$ at $1.5 \mathrm{~m}$ and $1.9 \%$ at $5.5 \mathrm{~m}$, and for Case 2 it was $0.2 \%$ at $1.5 \mathrm{~m}$ and $0.5 \%$ at $5.5 \mathrm{~m}$

The identified values of contraction parameters $c_{1}$ have a reasonable agreement with those used in the computational simulation; however, it is noted that differences between identified and actual parameters used in the simulation are of around $10 \%$. These discrepancies arise from the fact that vertical strains associated to volumetric changes that take place due to water flow and consolidation during the shaking are defined as zero in the identification analysis. Indeed, if the actual (synthetic) vertical strain information is used in the stress-strain model and identification, the exact actual value of $c_{1}$ is estimated. This is illustrated by Fig. 12(a), which shows an exact match between $\boldsymbol{\tau}^{(m)}(t)$ and $\boldsymbol{\tau}^{(r)}(t)$, and $\left|\Delta \sigma_{v}^{\prime}(t)\right|$ and $\Delta u_{w}(t)$, after introducing the complete strain time history (including vertical strains) to the constitutive model along with the actual value of $c_{1}(0.02)$, for Case 1 at a $5.5 \mathrm{~m}$ depth. Figure 12(b), on the other hand, shows the model prediction that is obtained using the actual value of $c_{1}$ if vertical strains are assumed to be zero. Relatively small discrepancies are evidenced between the synthetic deposit response and the model prediction used for the identification, with differences increasing after the soil excitation. It should be pointed out that vertical strains in the simulated deposit continue to increase after the end of shaking due to the consolidation (pore pressure dissipation) taking place in the 
sand. These vertical strains are associated to the decrease in excess pore water pressure observed in Fig. 12(b). In the absence of vertical strain information, the model used for the identification cannot capture the pore pressure dissipation after the end of shaking, leading to discrepancies increasing with time during the dissipation phase. It is evident, on the other hand, that inclusion of vertical strains results in an exact match of the stress conditions, since these strains implicitly provide information about the effects of water flow during and after excitation. For an analysis performed ignoring vertical strains, discrepancies are larger for soils with high permeability (which involve a greater flow of water). These discrepancies are less significant and eventually negligible for soils with lower permeabilities or lower contraction. In spite of the limitations of not having information on the vertical strains, identified values of $c_{1}$ still provide a reasonable estimation of the contractive behavior of the analyzed soils (i.e., soils with permeabilities on the order of 1e$5 \mathrm{~m} / \mathrm{s}$ or lower). 


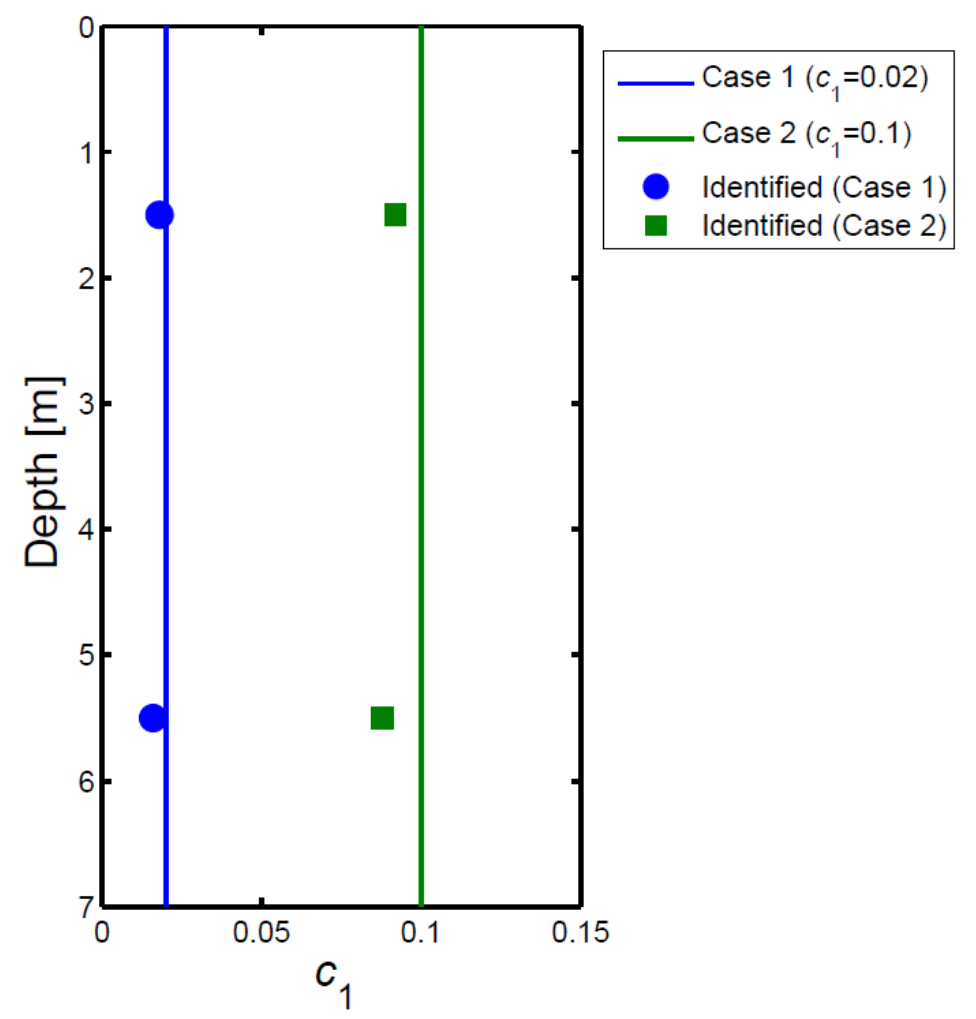

Fig. 10. Identified values of contraction parameter $c_{1}$ along with actual parameter profiles from the computational models. 

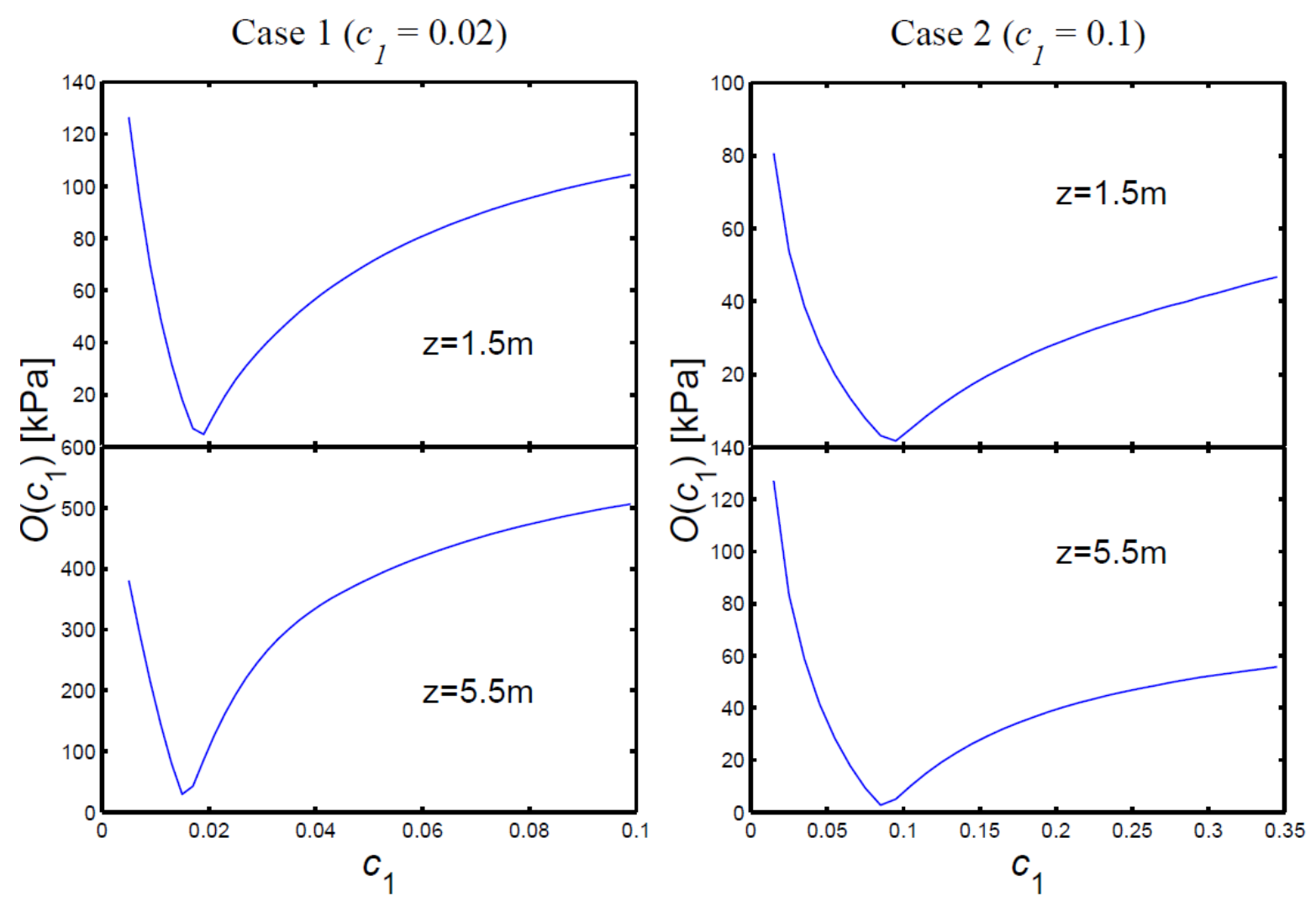

Fig. 11. Variation of the objective function $O\left(c_{1}\right)$ as a function of $c_{1}$ for Case 1 and Case 2 . 


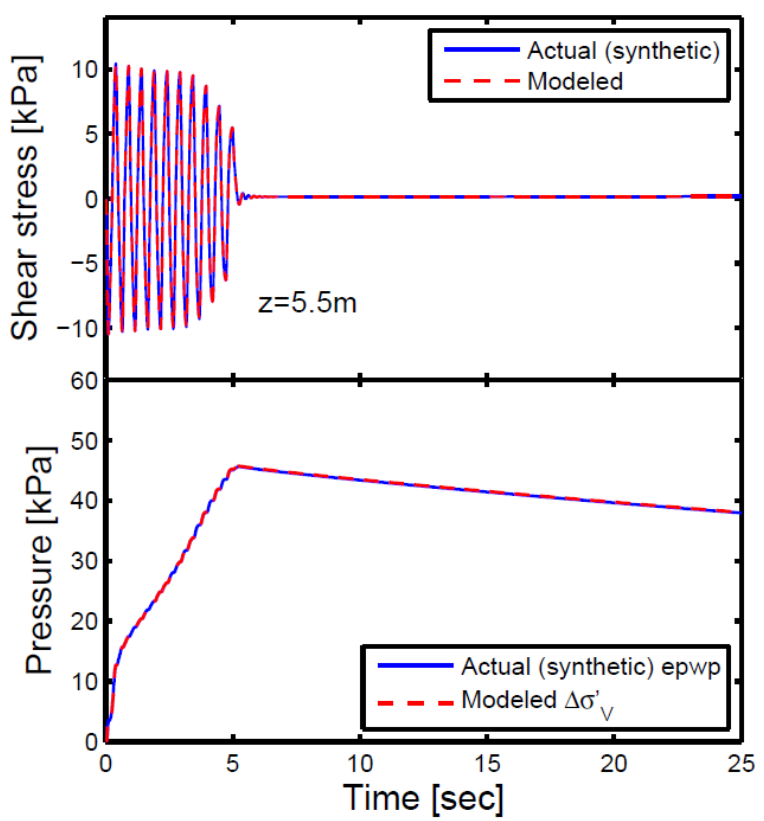

(a)

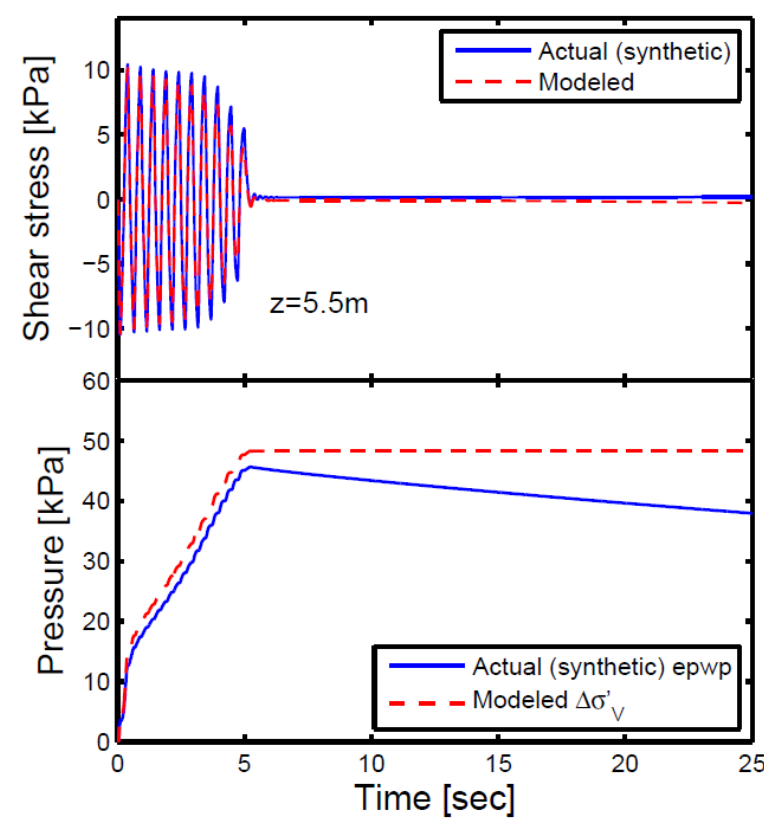

(b)

Fig. 12. Shear stress time histories comparison, and comparison of excess pore water pressure (epwp) and absolute value of the change in effective vertical stress $\left(\Delta \sigma^{\prime}{ }_{v}\right)$ between the computer simulation and the modeled response obtained: (a) using the complete strain time history (including vertical strains), and (b) imposing zero vertical strains.

Table 1. Summary of identification results for Case 1 and Case2.

\begin{tabular}{|c|c|c|}
\hline Case & $\begin{array}{c}\text { Identified } \boldsymbol{c}_{\mathbf{1}} \\
\text { at } \mathrm{z}=\mathbf{1 . 5 m}\end{array}$ & $\begin{array}{c}\text { Identified } \boldsymbol{c}_{\mathbf{1}} \\
\text { at } \mathrm{z}=\mathbf{5 . 5 m}\end{array}$ \\
\hline $\begin{array}{c}\text { Case 1 } \\
\left(\boldsymbol{c}_{\mathbf{1}}=\mathbf{0 . 0 2}\right)\end{array}$ & 0.018 & 0.016 \\
\hline $\begin{array}{c}\text { Case 2 } \\
\left(\boldsymbol{c}_{\mathbf{1}}=\mathbf{0 . 1}\right)\end{array}$ & 0.092 & 0.088 \\
\hline
\end{tabular}




\section{Analysis of experimentally measured deposit responses}

The developed technique was employed to analyze the measured responses of sand deposits in two experiments representative of liquefiable sand deposits: (1) a reduced-scale centrifuge test, and (2) a large full-scale test. Description of the tests and analysis of the results are described in the following subheadings.

\subsection{Centrifuge test}

A reduced-scale test of a saturated sand deposit was conducted in a laminar box under a 25 g gravity field using the centrifuge at Rensselaer Polytechnic Institute (RPI). The deposit was constructed using silty sand with a relative density of $30 \%$. This soil had $21 \%$ nonplastic fines content with a specific gravity of 2.69 and grain size distribution characterized by $\mathrm{D}_{10}=0.031 \mathrm{~mm}$ and $\mathrm{D}_{50}=0.103 \mathrm{~mm}$. The deposit was constructed using dry pluviation and saturated following the standard procedures implemented at RPI [8], [34]. The height of the soil model was $24 \mathrm{~cm}$, representing a prototype depth of $6 \mathrm{~m}$. Hereafter, prototype units are employed when discussing centrifuge experiment results. The input base excitation consisted of a nearly uniform sinusoidal acceleration with a $2 \mathrm{~Hz}$ frequency and a peak of approximately $0.02 \mathrm{~g}$. The response of the soil deposit was monitored using accelerometers, pore pressure transducers and bender elements. A schematic of the laminar container along with the configuration of the sensors used in the experiment is shown in Fig. 13. This is the same experiment labeled as Experiment 2 in [35]. 

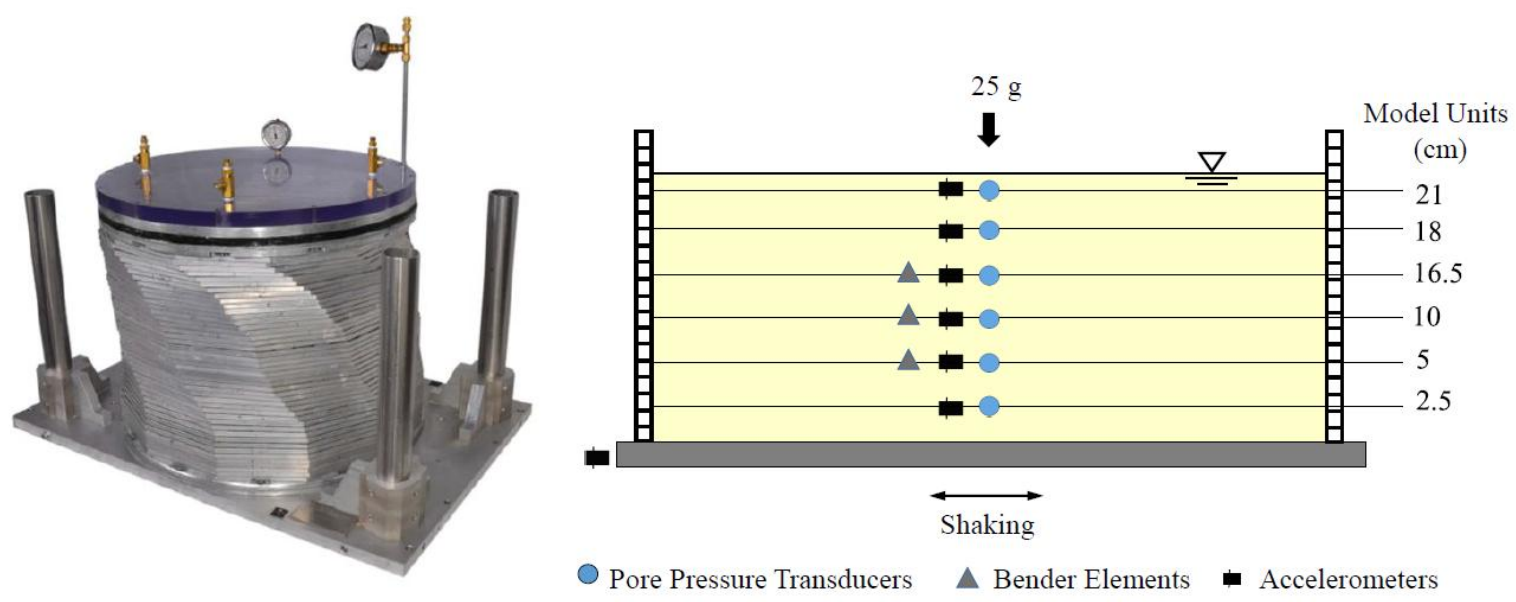

Fig. 13. Laminar container and configuration of the instrumentation used for the centrifuge test.

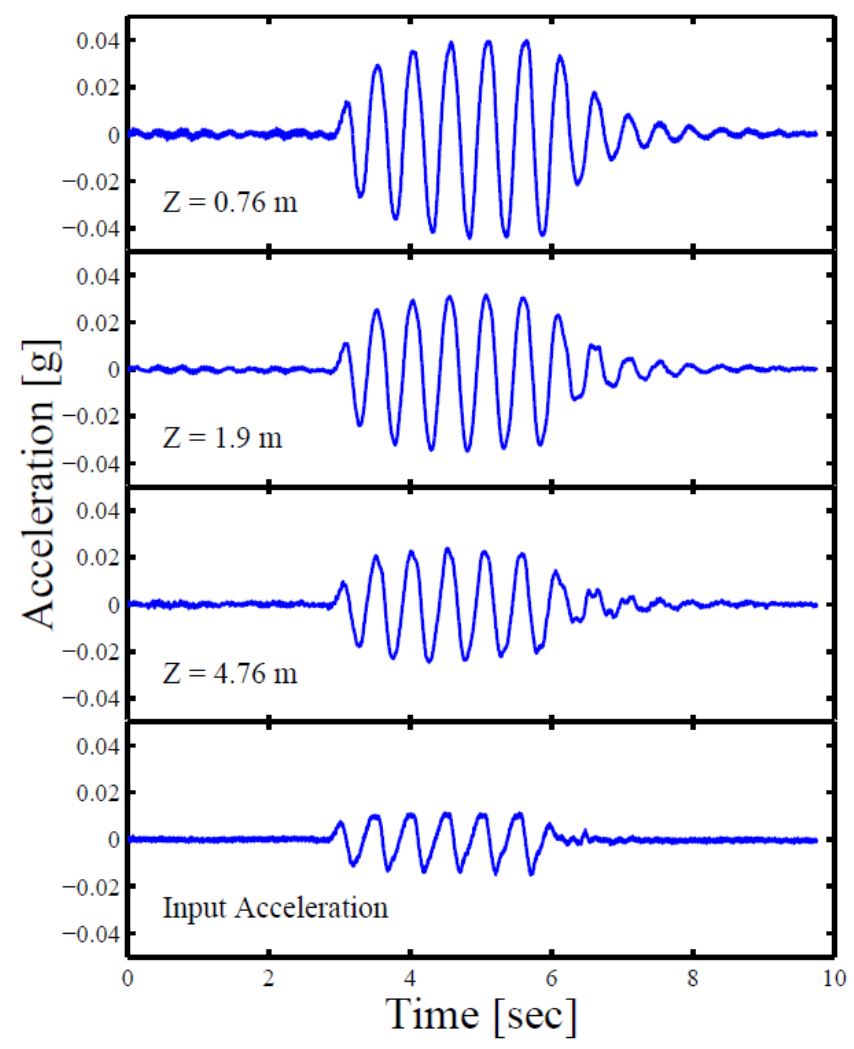

Fig. 14. Input and recorded soil accelerations at selected depth locations for centrifuge test. 
The accelerations recorded at the base and at selected locations within the deposits are displayed in Fig. 14. Shear stress-strain time histories were estimated using the recorded accelerations along with a corrected mass density of the system (including soil, water and laminar box rings), as described by Abdoun et al. [8]. A finite impulse response band-pass filter $(1.3 \mathrm{~Hz}$ to $5 \mathrm{~Hz}$ ) was employed to remove minor high and low frequency components in the processing of the acceleration records [36]. Values of $G_{\max }$ at the initial mean effective stress levels were inferred from direct shear wave velocity measurements obtained using bender elements [7]. Corresponding values of the reference shear deformation $\gamma_{\text {ref }}$ (at the initial mean effective stress levels) were obtained through a preliminary identification analysis using a technique proposed by Mercado et al. [22] for a $1 \mathrm{~s}$ time window $\left[t_{i}=3 \mathrm{~s} ; t_{f}=4 \mathrm{~s}\right.$ ]. This time window corresponds to the initiation of the shaking for which levels of generated excess pore pressure are small. Values of $G_{\max }$ and $\gamma_{\text {ref }}$ used in the subsequent identification analysis are summarized in Table 2 . It should be mentioned that the stress-strain model used in the proposed identification scheme is completely defined by parameters $G_{m a x}, \gamma_{r e f}$, and $c_{1}$. No explicit information is required on other soil properties such as permeability, grain size distribution or relative density of the soils.

Estimated shear stress and strain time histories are used along the recorded excess pore pressure response within the objective function (Eq. 11) at selected depths. As mentioned earlier, vertical strains are neglected in this process. The optimization analysis was performed for a $6 \mathrm{~s}$ time windows: $\left[t_{i}=3 \mathrm{~s} ; t_{f}=9 \mathrm{~s}\right]$. Minimization of the objective function $O\left(c_{1}\right)$ leads to the identified values of contraction parameter $c_{1}$ summarized in Table 2. Identified values of the contractive parameters along the depth of the deposit ranged from 0.03 to 0.07 , approximately. 
Table 2. Values of $G_{\max }$ and $\gamma_{\text {ref }}$ used in the identification analysis of the centrifuge test along with subsequently identified values of contraction parameter $c_{1}$.

\begin{tabular}{|c|c|c|c|}
\hline Depth $[\mathbf{m}]$ & $\boldsymbol{G}_{\boldsymbol{m a x}}[\mathbf{k P a}]$ & $\gamma_{\text {ref }}[\%]$ & Identified $\boldsymbol{c}_{\boldsymbol{1}}$ \\
\hline $\mathbf{1 . 3}$ & 6,053 & $2.13 \mathrm{E}-02$ & 0.046 \\
\hline $\mathbf{2 . 7}$ & 8,617 & $4.66 \mathrm{E}-02$ & 0.065 \\
\hline $\mathbf{4 . 1}$ & 10,611 & $3.99 \mathrm{E}-02$ & 0.028 \\
\hline $\mathbf{5 . 1}$ & 11,830 & $5.27 \mathrm{E}-02$ & 0.034 \\
\hline
\end{tabular}

Very close agreement is observed between the shear stress time histories estimated from recorded accelerations and those predicted by the material model using the identified material parameters (Fig. 15). The associated average relative error $E_{\tau}$ was of $15 \%$ for the estimated shear stress time histories. Recorded excess pore pressures are also in very close agreement with the magnitudes of the change in vertical effective stress, $\left|\Delta \sigma_{v}^{\prime}\right|$, predicted by the material model using the identified parameters, as shown in Fig. 16. The associated average relative error $E_{\sigma}$ was of $12 \%$ for the estimated time histories of change in vertical effective stress. Decreasing excess pore water pressures by the end of shaking at shallower depths is not very accurately captured by the model used in the identification since this would require information on the vertical strains associated to the pore pressure dissipation phenomena taking place in the sand. It is also possible that a more complex formulation of the constitutive laws dictating the volumetric behavior would yield a more accurate prediction of the increase in the excess pore water pressure. However, it is apparent that the 
employed constitutive model along with optimal material parameters is able to adequately capture the behavior of the deposit.

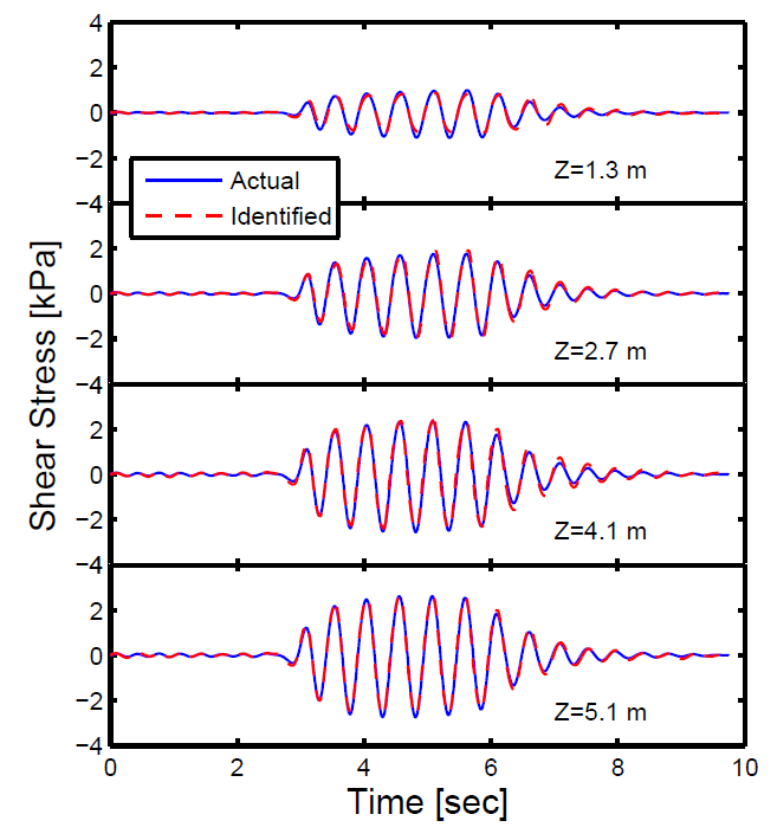

Fig. 15. Shear stress time histories estimated from acceleration records along with those obtained using the identified contraction parameter $c_{1}$ for the centrifuge test. 


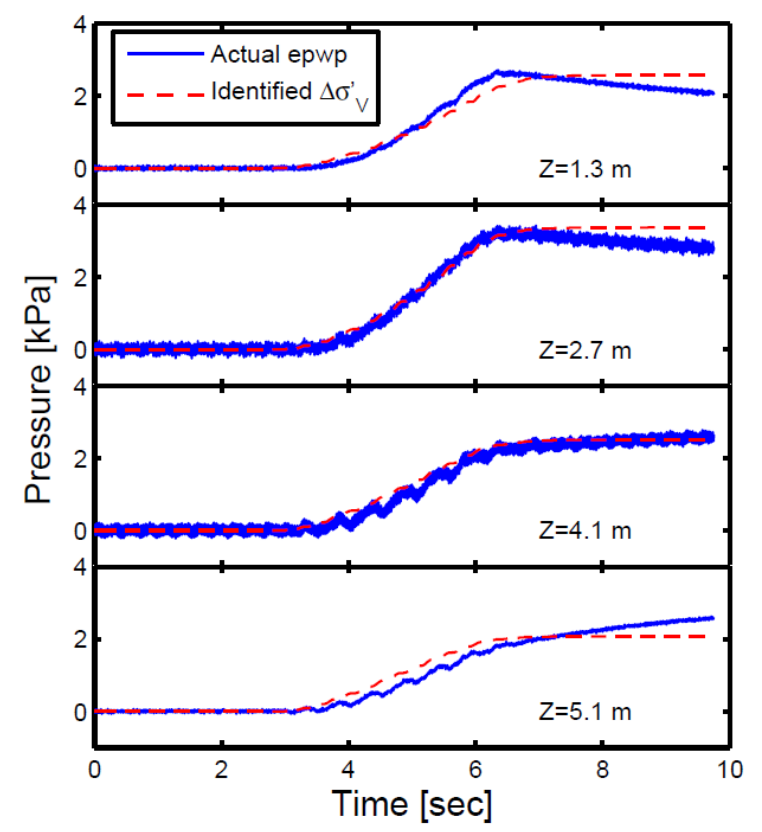

Fig. 16. Recorded excess pore water pressure (epwp) along with absolute value of the change in effective vertical stress $\left(\Delta \sigma^{\prime}{ }_{v}\right)$ obtained using the identified contraction parameter $c_{1}$ for the centrifuge test.

\subsection{Large-scale test}

A large-scale test of a saturated sand deposit was conducted in the large scale laminar container at the University at Buffalo (UB). The soil used in this experiment was clean Ottawa F\#55 sand which is characterized by being fine and uniform, and it has a specific gravity of 2.67. The minimum and maximum densities are $\rho_{\min }=1475 \mathrm{~kg} / \mathrm{m}^{3}$ and $\rho_{\max }=$ $1720 \mathrm{~kg} / \mathrm{m}^{3}$ [8]. The relative density of the soil deposit was about $40 \%$. 
The deposition method used was a simulation of hydraulic filling [37]. In this procedure, the sand is transferred in the form of slurry and pumped into the laminar container. The sand grains are allowed to settle by gravity in water very slowly in a manner similar to the deposition of sand in natural and man-made water bodies, which is known as alluvial deposition [37]. The large-scale model was instrumented with a variety of sensors to measure accelerations, pore pressures, and vertical and horizontal deformations, as shown in Fig. 17. This is the same experiment labeled as Experiment 4 in [35].
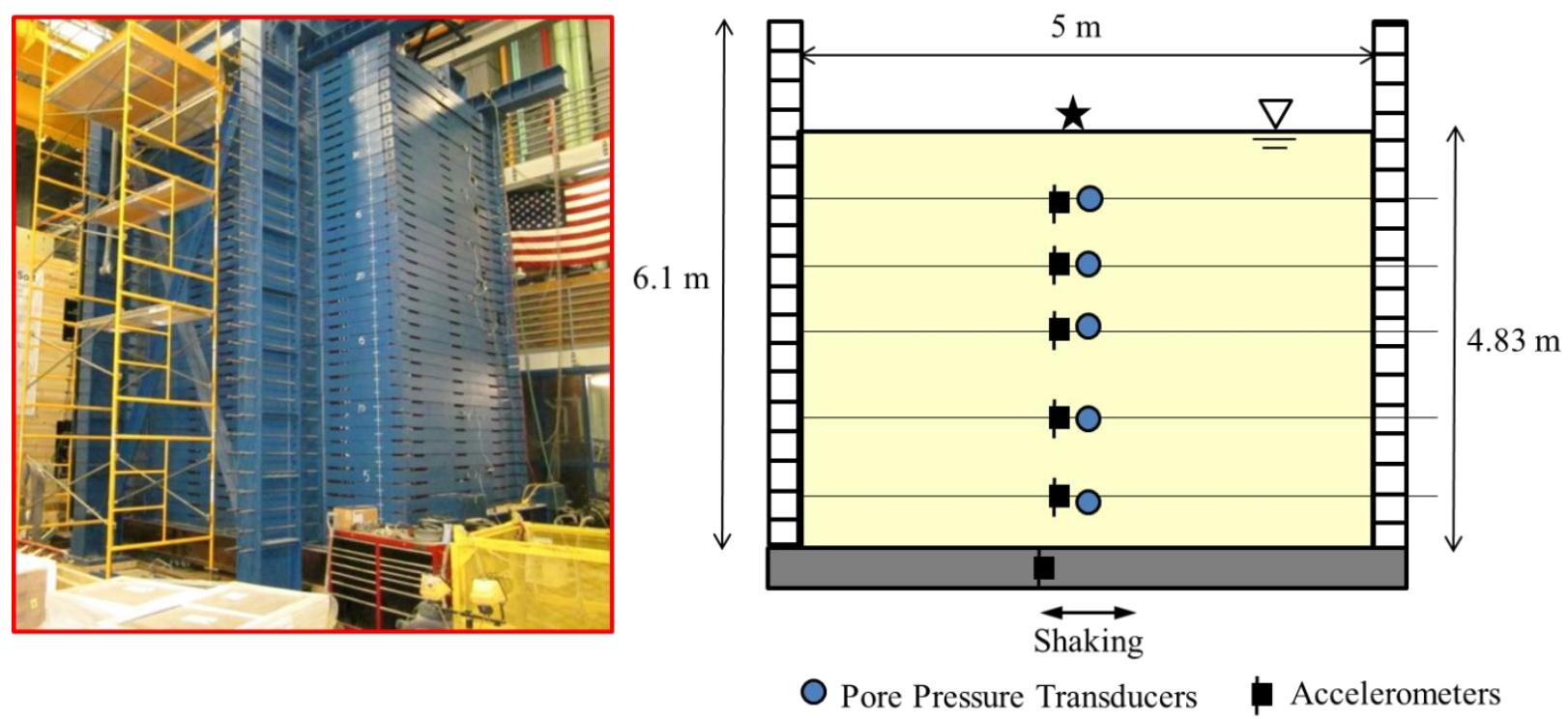

Vertical Potentiometer

Fig. 17. Laminar container and configuration of the instrumentation used for the large-scale test. 


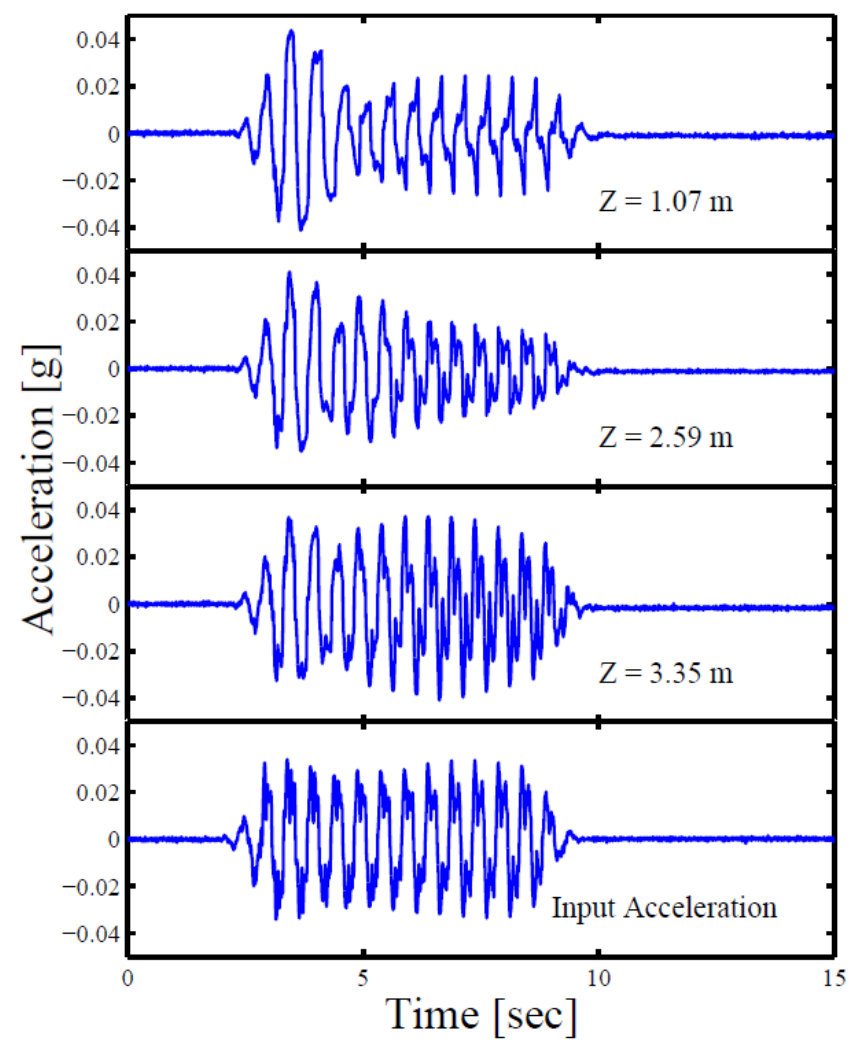

Fig. 18. Input and recorded soil accelerations at selected depth locations for large-scale test.

Figure 18 shows the accelerations recorded at the base and at selected locations within the deposit. Shear stress-strain time histories were estimated from the recorded accelerations using the same procedures employed in the analysis of the reduced-scale centrifuge test. Values of $G_{\max }$ at the initial mean effective stress levels were inferred from system identification using a weak shaking applied to the system just before the main shaking. For the estimation of $\gamma_{\text {ref }}$, a preliminary identification analysis [22] was performed for a $1 \mathrm{~s}$ time window $\left[t_{i}=2 \mathrm{~s} ; t_{f}=3 \mathrm{~s}\right.$ ], corresponding to the initiation of shaking for which levels of generated excess pore pressure are reasonably small. Values of $G_{\max }$ and $\gamma_{\text {ref }}$ used for the identification analysis are summarized in Table 3. 
Table 3. Values of $G_{\max }$ and $\gamma_{\text {ref }}$ used in the identification analysis of the large-scale test along with subsequently identified values of contraction parameter $c_{1}$.

\begin{tabular}{|c|c|c|c|}
\hline Depth $[\mathbf{m}]$ & $\boldsymbol{G}_{\boldsymbol{m a x}}[\mathbf{k P a}]$ & $\gamma_{\text {ref }}[\%]$ & Identified $\boldsymbol{c}_{\boldsymbol{I}}$ \\
\hline $\mathbf{1 . 4}$ & 11,059 & $1.16 \mathrm{E}-02$ & 0.38 \\
\hline $\mathbf{2 . 2}$ & 13,653 & $1.21 \mathrm{E}-02$ & 0.32 \\
\hline $\mathbf{3 . 0}$ & 15,828 & $2.41 \mathrm{E}-02$ & 0.37 \\
\hline $\mathbf{4 . 0}$ & 18,277 & $2.35 \mathrm{E}-02$ & 0.32 \\
\hline
\end{tabular}

The identification analysis was performed similarly to the analysis of the reducedscale deposit, using an $8 \mathrm{~s}$ time windows: $\left[t_{i}=2 \mathrm{~s} ; t_{f}=10 \mathrm{~s}\right]$. The identified contraction parameters $c_{1}$ are summarized in Table 3 and depicted in Fig. 21. Values of the contractive parameters are fairly constant along the depth of the deposit ranging from approximately 0.3 to 0.4 .

As can be seen in Figs. 19 and 20, the implemented constitutive model is able to accurately predict the soil response up to approximately 5 to $6 \mathrm{~s}$ (for the upper depths). At these times, identified values of the vertical effective stresses are reduced to the minimum value allowed in the model (values close to zero), and the prediction has to be terminated. Reaching a value of zero for the vertical effective stress indicates the initiation of liquefaction at a given location (this does not mean that the full deposit failed, as soil at neighboring locations may be not fully liquefied). The identified soil response in Figs. 19 and 20 is shown only up to the initiation of liquefaction since the implemented model 
cannot capture the post-liquefaction behavior of the deposit, including possible dilation, residual shear stresses and permanent displacements. Other soil constitutive models (such as models by Elgamal et al. [33], or by Dafalias and Manzari [38]) could be used along with the technique presented hereby in order to identify the cyclic liquefaction response of soil deposits. Implementation of such models would imply the calibration of additional parameters associated to the post-liquefaction behavior of soils.

A relatively good agreement is observed between the shear stress time histories estimated from recorded accelerations and those predicted by the material model using the identified material parameters (Fig. 19). The associated average relative error $E_{\tau}$ ranged between $25 \%$ and $35 \%$ for the estimated shear stress time histories. Peak values identified shear stresses are consistently slightly smaller than the actual for all considered depths. Discrepancies between identified and estimated shear stresses are mostly due to the inability of the selected stress-strain relation (Eq. 7) to accurately describe the strain dependent stress response. The implemented hyperbolic stress-strain relation was selected because of its simplicity since it only depends on two parameters $\left(G_{\max }\right.$ and $\left.\gamma_{\text {ref }}\right)$; however, it has been noticed that it cannot always accurately capture the soil stress-strain behavior [38], [39]. Other stress-strain relations might yield a better description of the soil response. For instance, the MKZ model [38] introduces two additional fitting parameters into the stress-strain definition leading to a more accurate description of the stress response, but adopting such a model would add greater complexity to the problem since it would imply calibrating for more variables. Recorded excess pore pressures, on the other hand, also show a good agreement with the magnitudes of the change in vertical effective stress, $\left|\Delta \sigma_{v}^{\prime}\right|$, predicted by the material model using the identified parameters, as shown in Fig. 20. The associated average relative error $E_{\sigma}$ ranged between $9 \%$ and $20 \%$ for the estimated 
time histories of change in vertical effective stress. The employed constitutive model along with optimal material parameters is able to adequately capture the behavior of the deposit up to the initiation of liquefaction.

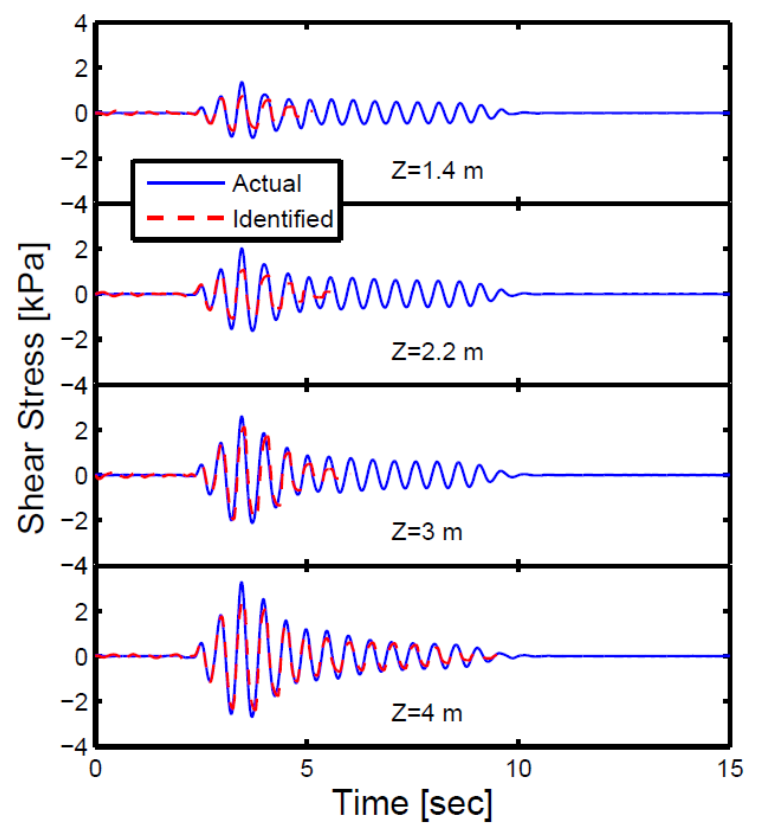

Fig. 19. Shear stress time histories estimated from acceleration records along with those obtained using the identified contraction parameter $c_{1}$ for the centrifuge test. 


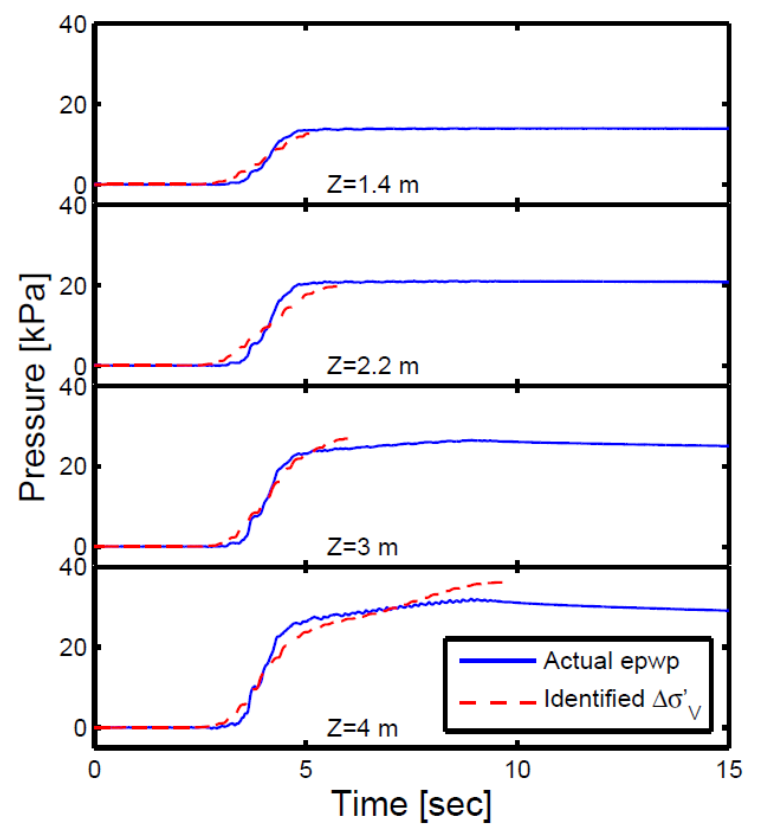

Fig. 20. Recorded excess pore water pressure (epwp) along with absolute value of the change in effective vertical stress $\left(\Delta \sigma^{\prime}{ }_{\mathrm{v}}\right)$ obtained using the identified contraction parameter $c_{1}$ for the large-scale test. 


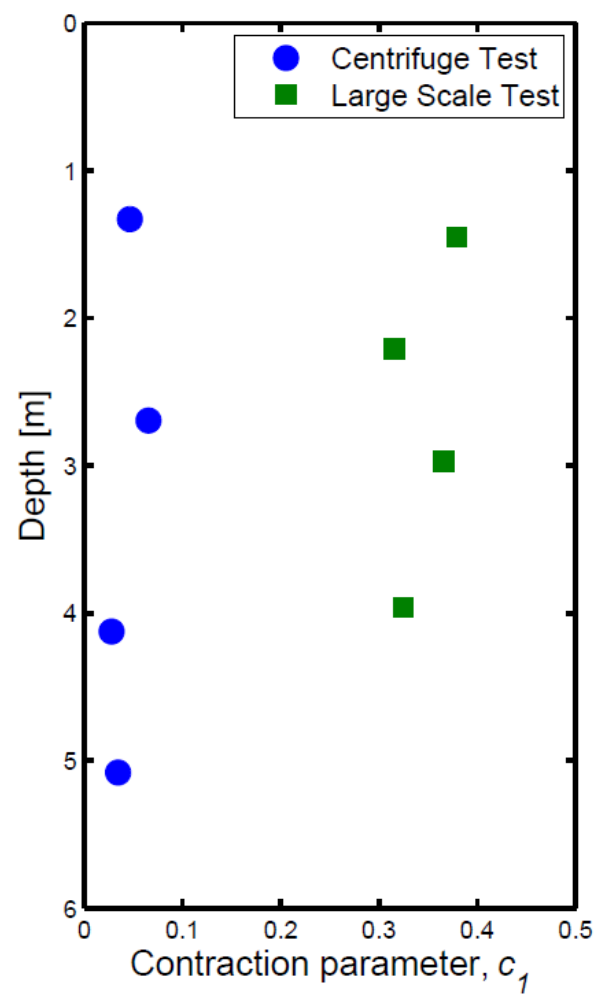

Fig. 21. Identified values of contraction parameter $c_{1}$ for the centrifuge test and the largescale test.

\subsection{Comparison between centrifuge test and large-scale test}

Identified values of $c_{1}$ along the depth of the centrifuge deposit ranged from 0.03 to 0.07 , approximately, whereas identified values of $c_{1}$ for the large scale deposit ranged from approximately 0.3 to 0.4 . These values are comparable with suggested ranges by Yang et al. [39], which propose values of $c_{1}=0.03$ for looser sands, and $c_{1}=0.2$ for denser sands. Elgamal et al. [33], for instance, report a value of $c_{1}=0.17$ for Nevada sand at a relative density of about $40 \%$. 
The difference between the ranges of identified $c_{1}$ values for the two analyzed deposits is consistent with the observed behavior of the recorded acceleration and pore pressure build up: the higher values of $c_{1}$ in the large scale deposit are accompanied by a higher rate of pore water pressure buildup and a marked decrease in the amplitude of the shear stress peaks. Lower values of $c_{1}$ identified in the centrifuge deposit, on the other hand, are consistent with the low levels of pore pressure build up and relatively uniform shear stress behavior.

The difference between values of $c_{1}$ for the hydraulically filled clean sand in the large scale test and the dry pluviated silty sand in the centrifuge test is of almost one order of magnitude (Fig. 21). This is significant and consistent with the greater rate of pore pressure build up developed in Test SG-1 (hydraulically filled) compared to Test FF-P2 (dry pluviated) in Abdoun et al. [8]. This finding is consistent with the observation that a hydraulic fill deposit often has a fabric that is more collapsible (i.e, more contactive) and leads to larger excess pore pressure build up than a dry pluviated sand deposit for the same relative density, and same level of applied confining stresses and shear strain [40]. As stated by Abdoun et al.: "The fact that the deposition method or fabric may have a dramatic effect on pore pressure response during cyclic stress-controlled loading has long been known from results on small-sample cyclic laboratory tests [41] [42] [43].”

\section{Conclusions}

This paper presented an identification technique to estimate the coupled shear-volume response leading to pore pressure generation in a contractive soil deposit subjected to 
lateral excitations. The technique uses acceleration and pore pressure records provided by vertical (downhole) arrays of accelerometers and pore pressure sensors. The proposed methodology provides estimates of the parameters defining the contractive tendency of the soil along the zone instrumented by the vertical array. The stress-strain relation is modeled using a multi-surface plasticity approach. The volumetric (contractive) behavior of the soil is defined in terms of a single contraction parameter, $c_{1}$. Identification of the optimal contractive parameters is carried out through the minimization of an objective function which is evaluated based on discrepancies between the modeled stress response, and recorded pore pressure build up and non-parametric estimates of the shear stresses derived from recorded accelerations. Consequently, the forward modeling of the whole soil deposit is not required. It should be noted that different soil constitutive models can be used along with this technique in order to accommodate more complex behavior including, for instance, the dilative response of soils.

The technique was verified using numerical simulations and implemented using data of a reduced-scale centrifuge test and a large scale experiment. The centrifuge test consisted of a level site deposit of a dry pluviated silty sand, and the large scale experiment consisted on a hydraulically filled clean sand deposit. The implemented material model was able to adequately capture the stress-strain behavior of the deposits after a proper identification of the optimal material parameters. Recorded pore pressure build up was in very good agreement with the modeled reduction of vertical effective stress for both tests.

The proposed technique proved to be useful as an effective means to analyze the dynamic soil behavior based on the recorded response at a site. Through the integration of acceleration information, pore pressure data and numerical modeling, this methodology allows for a more complete interpretation of the coupled shear-volume response of a soil 
profile. Results of the identification can be used in the analysis of the pore pressure buildup, as well as in the prediction of site response during future events.

\section{Acknowledgements}

This paper is based on work supported by the National Science Foundation under NEESRSG Grant No. 0529995, and by Universidad del Norte. This support is greatly appreciated.

\section{References}

[1] I. Idriss and R. Boulanger, "Soil liquefaction during earthquakes," Earthquake engineering research institute, 2008.

[2] M. Zeghal and A. Elgamal, "Analysis of site liquefaction using earthquake records," Journal of geotechnical engineering, vol. 120, no. 6, pp. 996-1017, 1994.

[3] A. Elgamal, "Dynamic soil properties, seismic downhole arrays and applications in practice," in International conference on recent advances in geotechnical earthquake and soil dynamics, 2001.

[4] J. Steidl, S. Seale, R. Gee, H. Ratzesberger and V. Gorbunov, "Permanently Instrumented Field Sites Database, UCSB," Network for Earthquake Engineering Simulation (database), Dataset, DOI:10.4231/D3XP6V349, 2010.

[5] D. Assimaki, W. Li and A. Kalos, "A wavelet-based seismogram inversion algorithm for the in situ characterization of nonlinear soil behavior," Pure and applied geophysics, vol. 168, no. 10, pp. 1669-1691, 2011.

[6] D. R. Groholski, Y. M. Hashash and N. Matasovic, "Learning of pore pressure response and dynamic soil behavior from downhole array measurements," Soil Dynamics and Earthquake Engineering, 61, 40-56., vol. 61, pp. 40-56, 2014. 
[7] W. El-Sekelly, A. Tessari and T. Abdoun, "Shear wave velocity measurement in the centrifuge using bender elements," Geotechnical Testing Journal, vol. 37, no. 4, pp. 689-704., 2014.

[8] T. Abdoun, M. Gonzalez, S. Thevanayagam, R. Dobry, A. Elgamal, M. Zeghal, V. Mercado and U. El Shamy, "Centrifuge and large-scale modeling of seismic pore pressures in sands: Cyclic strain interpretation," Journal of Geotechnical and Geoenvironmental Engineering, vol. 139, no. 8, pp. 1215-1234, 2013.

[9] C. J. Lee, Y. C. Wei and Y. C. Kuo, "Boundary effects of a laminar container in centrifuge shaking table tests," Soil Dynamics and Earthquake Engineering, vol. 34, no. 1, pp. 37-51, 2012.

[10] R. Dobry, S. Thevanayagam, C. Medina, R. Bethapudi, A. Elgamal, V. Bennett, T. Abdoun, M. Zeghal, U. El Shamy and V. Mercado, "Mechanics of lateral spreading observed in a full-scale shake test," Journal of geotechnical and geoenvironmental engineering, vol. 137, no. 2, pp. 115-129, 2010.

[11] C. C. Tsai, W. C. Lin and J. S. Chiou, "Identification of dynamic soil properties through shaking table tests on a large saturated sand specimen in a laminar shear box," Soil Dynamics and Earthquake Engineering, vol. 83, pp. 59-68, 2016.

[12] M. Zeghal, A. W. Elgamal, H. T. Tang and J. C. Stepp, "Lotung downhole array. II: Evaluation of soil nonlinear properties," Journal of geotechnical engineering, vol. 121, no. 4, pp. 363-378, 1995.

[13] D. Assimaki, J. Steidl and P. C. Liu, "Attenuation and velocity structure for site response analyses via downhole seismogram inversion," Pure and applied geophysics, vol. 163, no. 1, pp. 81-118, 2006.

[14] D. Assimaki and J. Steidl, "Inverse analysis of weak and strong motion downhole array data from the M w 7.0 Sanriku-Minami earthquake," Soil Dynamics and Earthquake Engineering, vol. 27, no. 1, pp. 73-92, 2007.

[15] D. Assimaki, W. Li, J. H. Steidl and K. Tsuda, "Site amplification and attenuation via downhole array seismogram inversion: A comparative study of the 2003 Miyagi-Oki aftershock sequence," Bulletin of the Seismological Society of America, vol. 98, no. 1, pp. 301-330, 2008.

[16] C. C. Tsai and Y. M. Hashash, "A novel framework integrating downhole array data and site response analysis to extract dynamic soil behavior," Soil Dynamics and Earthquake Engineering, vol. 28, no. 3, pp. 181-197, 2008. 
[17] C. C. Tsai and Y. M. Hashash, "Learning of dynamic soil behavior from downhole arrays," Journal of Geotechnical and Geoenvironmental Engineering, vol. 135, no. 6, pp. 745-757, 2009.

[18] N. Matasovic, "Seismic response of composite horizontally-layered soil deposits," University of California, Los Angeles, 1993.

[19] Z. Yang and A. Elgamal, "Application of unconstrained optimization and sensitivity analysis to calibration of a soil constitutive model," International journal for numerical and analytical methods in geomechanics, vol. 27, no. 15, pp. 1277-1297, 2003.

[20] D. R. Groholski and Y. Hashash, "Development of an inverse analysis framework for extracting dynamic soil behavior and pore pressure response from downhole array measurements," International Journal for Numerical and Analytical Methods in Geomechanics, vol. 37, no. 12, pp. 1867-1890, 2013.

[21] J. H. Prevost, "A simple plasticity theory for frictional cohesionless soils," International Journal of Soil Dynamics and Earthquake Engineering, vol. 4, no. 1, pp. 9-17, 1985.

[22] V. Mercado, W. El-Sekelly, M. Zeghal and T. Abdoun, "Identification of soil dynamic properties through an optimization analysis," Computers and Geotechnics, vol. 65, pp. 175-186, 2015.

[23] Z. Mroz, "On the description of anisotropic workhardening," Journal of the Mechanics and Physics of Solids, vol. 15, no. 3, pp. 163-175, 1967.

[24] J. H. Prevost, "Modeling the behavior of geomaterials," in Sayed SM, editor. Geotechnical modeling and applications, Gulf Publishing Company, 1987.

[25] Z. Yang, A. Elgamal and E. Parra, "Computational model for cyclic mobility and associated shear deformation," Journal of Geotechnical and Geoenvironmental Engineering, vol. 129, no. 12, pp. 1119-1127, 2003.

[26] R. L. Kondner, "Hyperbolic stress-strain response: cohesive soils," Journal of the Soil Mechanics and Foundations Division, vol. 89, no. 1, pp. 115-144., 1963.

[27] J. M. Duncan and C. Y. Chang, "Nonlinear analysis of stress and strain in soils," Journal of the soil mechanics and foundations division, vol. 96, no. 5, pp. 1629-1653, 1970. 
[28] F. H. Kulhawy and J. M. Duncan, "Stresses and movements in Oroville dam," Journal of Soil Mechanics \& Foundations Division, vol. 98, no. sm7, 1972.

[29] B. Hardin and V. P. Drnevich, "Shear modulus and damping in soils," Journal of the Soil Mechanics and Foundations Division, vol. 98, no. 7, pp. 667-692, 1972.

[30] E. J. Parra-Colmenares, "Numerical modeling of liquefaction and lateral ground deformation including cyclic mobility and dilation response in soil systems," Rensselaer Polytechnic Institute, Doctoral dissertation, Troy, NY, 1996.

[31] K. Ishihara, F. Tatsuoka and S. Yasuda, "Undrained deformation and liquefaction of sand under cyclic stresses," Soils and foundations, vol. 15, no. 1, pp. 29-44, 1975.

[32] The MathWorks Inc., "Optimization toolbox ${ }^{\mathrm{TM}}$ user's guide," Natick, MA, 2014.

[33] A. Elgamal, Z. Yang and E. Parra, "Computational modeling of cyclic mobility and post-liquefaction site response," Soil Dynamics and Earthquake Engineering, vol. 22, no. 4, pp. 259-271, 2002.

[34] M. A. Gonzalez, "Centrifuge modeling of pile foundation response to liquefaction and lateral spreading: Study of sand permeability and compressibility effects using scaled sand technique," Ph.D. dissertation, Rensselaer Polytechnic Institute, Troy, NY, 2008.

[35] W. El-Sekelly, "The effect of seismic preshaking history on the liquefaction resistance of granular soil deposits," Ph.D. dissertation, Rensselaer Polytechnic Institute, Troy, NY, 2014.

[36] A. Elgamal, M. Zeghal, V. Taboada and R. Dobry, "Analysis of site liquefaction and lateral spreading using centrifuge testing records," Soils and Foundations, vol. 36, no. 2, pp. 111-121, 1996.

[37] S. Thevanayagam, T. Kanagalingam, A. Reinhorn, R. Tharmendhira, R. Dobry, M. Pitman, T. Abdoun, A. Elgamal, M. Zeghal, N. Ecemis and U. El Shamy, "Laminar box system for 1-g physical modeling of liquefaction and lateral spreading," Geotechnical Testing Journal, vol. 32, no. 5, pp. 1-12, 2009.

[38] Y. F. Dafalias and M. T. Manzari, "Simple plasticity sand model accounting for fabric change effects," Journal of Engineering mechanics, vol. 130, no. 6, pp. 622-634, 2004.

[39] N. Matasovic and M. Vucetic, "Cyclic characterization of liquefiable sands," Journal of Geotechnical Engineering, vol. 119, no. 11, pp. 1805-1822, 1993. 
[40] I. M. Idriss, R. D. Singh and R. Dobry, "Nonlinear Behavior of Soft Clays during Cyclic Loading," Journal of the Geotechnical Engineering Division, vol. 104, no. 12, pp. 1427-1447, 1978.

[41] Z. Yang, J. Lu and A. Elgamal, "OpenSees Soil Models and Solid-Fluid Fully Coupled Elements," University of California, San Diego, 2008.

[42] M. Zeghal and C. Tsigginos, "A micromechanical analysis of the effect of fabric on low-strain stiffness of granular soils," Soil Dynamics and Earthquake Engineering, vol. 70, pp. 153-165, 2015.

[43] R. Ladd, "Specimen preparation and cyclic stability of sands," Journal of Geotechnical and Geoenvironmental Engineering, vol. 103, no. 6, pp. 535-547, 1977.

[44] B. Seed, "Soil liquefaction and cyclic mobility evaluation for level ground during earthquakes," Journal of Geotechnical and GEoenvironmental Engineering, vol. 105, no. 2, pp. 201-255, 1979.

[45] R. Dobry, R. S. Ladd, F. Y. Yokel, R. M. Chung and D. Powell, "Prediction of pore water pressure buildup and liquefaction of sands during earthquakes by the cyclic strain method," NBS Building Science Series 138, National Bureau of Standards, Gaithersburg, MD, 1982. 